\title{
A method for the simultaneous determination of transport and structural parameters of forward osmosis membranes
}

\author{
Alberto Tiraferri, Ngai Yin Yip, Anthony P. Straub, Santiago Romero-Vargas Castrillon, \\ Menachem Elimelech* \\ Department of Chemical and Environmental Engineering, Yale University, New Haven, CT 06520-8286, United States
}

\section{A R T I C L E I N F O}

\section{Article history:}

Received 17 March 2013

Received in revised form 2 May 2013

Accepted 6 May 2013

Available online 23 May 2013

Keywords:

Forward osmosis

Membrane characterization

Transport

Structural parameter

Active layer

Permeability

\begin{abstract}
A B S T R A C T
We present a simple and rapid methodology to characterize the water and solute permeability coefficients ( $A$ and $B$, respectively) and structural parameter $(S)$ of forward osmosis (FO) membranes. The methodology comprises a single FO experiment divided into four stages, each using a different concentration of draw solution. The experimental water and reverse salt fluxes measured in each stage are fitted to the corresponding FO transport equations by performing a least-squares non-linear regression, using $A, B$, and $S$ as regression parameters. Hand-cast thin-film composite (TFC) FO membranes and commercial TFC FO, TFC reverse osmosis (RO), and cellulose acetate-based asymmetric FO membranes are evaluated following this protocol. We compare the membrane properties obtained with our FO-based methodology with those derived from existing protocols based on an RO experiment followed by an FO experiment. For all membranes, the FO-based protocol gives more accurate predictions of the water and salt fluxes than the existing method. The numerical robustness of the method and the sensitivity of the regression parameters to random errors in the measured quantities are thoroughly analyzed. The assessment shows that confidence in the accuracy of the determined membrane parameters can be enhanced by simultaneously achieving close fitting of the predicted fluxes to experimental measurements (i.e., high $R^{2}$ values) and constant water to salt flux ratios in each stage. Additionally, the existing and proposed approaches yield consistently dissimilar results for some of the analyzed membranes, indicating a discrepancy that might be attributed to the different driving forces utilized in RO and in FO that should be further investigated.
\end{abstract}

(c) 2013 Elsevier B.V. All rights reserved.

\section{Introduction}

Forward osmosis (FO) utilizes the osmotic pressure difference developed across a semi-permeable membrane separating two solutions of different concentrations to drive the permeation of water [1]. FO has shown promise in a variety of applications [1-7], and it is also attracting attention as a potential technology to augment water supplies using seawater $[1,8,9]$ and wastewater [10-12]. Where abundant and low value streams can be used without the need for regeneration, such as seawater and wastewater, FO can be employed to concentrate the feed solution (osmotic concentration) or dilute the draw solution (osmotic dilution) [13].

In recent years, a great deal of research has been directed at the fabrication of FO membranes [7,14-29]. These efforts have resulted in the development of substantially improved membranes tailored for the specific needs of FO. In particular, thin-film composite (TFC)

\footnotetext{
* Corresponding author. Tel.: +1 203432 2789; fax: +1 2034324387.

E-mail address: menachem.elimelech@yale.edu (M. Elimelech).
}

FO membranes, consisting of a salt-rejecting, active layer and a porous support, have shown higher water fluxes, reduced salt passage, and enhanced anti-fouling properties [22,29-31]. A convenient and consistent methodology to characterize FO membranes is of critical importance to advance this technology onto its mature phase, facilitating the sharing of data, their interpretation, and comparison.

When describing membrane performance, the literature often reports values of water fluxes, $J_{\mathrm{w}}$, reverse solute fluxes, $J_{\mathrm{s}}$, the resistance to solute diffusion in the membrane support layer, $K$, or its inverse parameter, the mass transfer coefficient, $k=1 / K$ [32]. However, these quantities are not intrinsic properties of the membrane as they depend on the hydrodynamic conditions at the membrane interface, the concentration and osmotic pressure of the draw and feed solutions, and the type and diffusivity of the solutes. This approach therefore lacks generality, as direct comparisons cannot be made unless the operating conditions are identical.

An alternative approach, adopted for 'tight', salt-rejecting FO membranes, is based on three intrinsic parameters that fully describe membrane systems: the pure water permeability 
coefficient, $A$, and the solute permeability coefficient, $B$, which describe the transport across the membrane active layer, and the structural parameter, $S$, quantifying the mass transport length scale across the membrane support layer. These three parameters are univocal and can be used with the respective governing equations to accurately predict the water and salt flux performance of a membrane sample in any laboratory-scale FO system. Therefore, the values of $A, B$, and $S$ represent common yardsticks for describing membrane intrinsic characteristics and offer a universal set of criteria for comparing performance, regardless of operating conditions.

The existing approaches to measure $A, B$, and $S$ of an FO membrane entail the use of at least two separate experiments. Initially, the parameters related to the active layer $(A$ and $B)$ are measured by applying a trans-membrane hydraulic pressure in reverse osmosis (RO) mode experiments. Subsequently, the membrane is tested using an osmotic driving force $[7,15,17-29,33]$ to determine the support layer structural parameter, $S$. Experiments in the pressure retarded osmosis (PRO) configuration (draw solution facing the active side of the membrane) may also be conducted to complement [17-19,21,24-27] or substitute [14] measurements in FO configuration.

These protocols are cumbersome and laborious, requiring multiple experiments in different experimental setups. Subjecting FO membranes, intended for operation near ambient conditions, to the high pressures typical of RO tests, can result in mechanical damage to the membrane. Furthermore, current methodologies combining RO and FO are based on the notion that transport parameters are universally valid and transferable, an assumption that warrants further examination in light of the fundamentally different permeation driving forces in RO and FO: a hydraulic pressure difference applied on the RO membrane active layer versus the osmotic pressure difference across the membrane active/support layer interface in FO. These fundamental differences may result in dissimilar observed transport parameters between the RO and FO processes, a phenomenon also suggested in recent studies [34]. It is therefore desirable to formulate a methodology for FO membrane characterization that evaluates the membrane performance under representative driving force and operating conditions, and which, in addition, is both simple (i.e., based on a minimum number of experiments) and reliable.

In this study, we present a method to characterize the intrinsic transport and structural properties of FO membranes in a single FO experiment. By changing the concentration of the draw solution in each stage of the experiment, a set of FO water flux and reverse salt flux measurements are obtained. Membrane parameters are determined through non-linear regression, where $A, B$, and $S$ are treated as adjustable parameters to fit the FO transport equations to the experimental water and salt fluxes. To demonstrate the generality of the method, we characterized four sets of membranes exhibiting a wide range of transport and structural parameters. Our results raise questions about the reliability of current membrane characterization protocols, and point towards further investigations in transport processes in osmotically driven membrane processes.

\section{A single FO experiment to characterize osmotic membranes}

A single and facile FO experiment is proposed to characterize the intrinsic transport parameters, $A$ and $B$, and the structural parameter, $S$, of an FO membrane by measuring the water and reverse solute flux across the membrane under different draw solution concentrations. As depicted schematically in Fig. 1, the FO experiment is divided into a discrete number of stages. The influence of the adopted number of stages on the robustness and accuracy of the methodology will be discussed in Section 5. In our study, the experiments were carried out in four stages.

In the first stage of the experiment, a draw solution concentration $c_{\mathrm{D}, 1}$ and a feed solution of deionized (DI) water were utilized to measure the FO water flux, $J_{\mathrm{w}, 1}$, and the reverse solute flux, $J_{\mathrm{s}, 1}$. At the end of the first stage, a known volume of concentrated draw

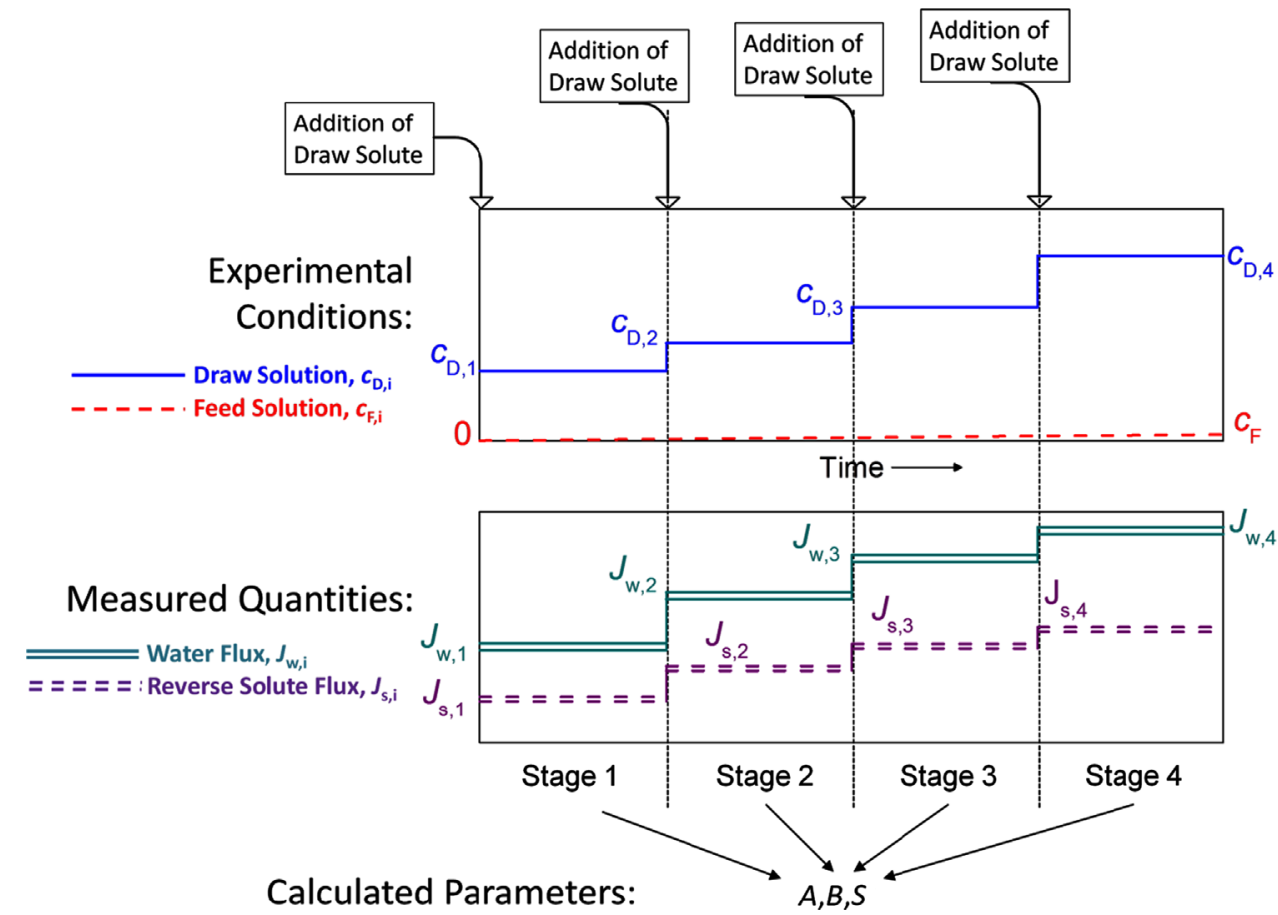

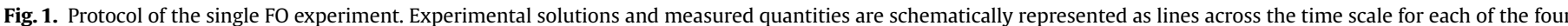

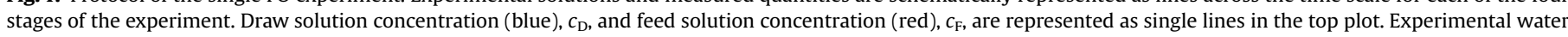

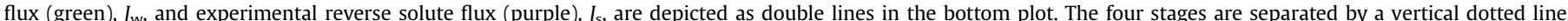

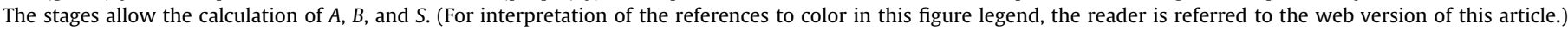


solution was added to increase the draw solution concentration from $c_{\mathrm{D}, 1}$ to $c_{\mathrm{D}, 2}$. The osmotic pressure and salt concentration difference across the membrane increased and, as a result, both the FO water flux and the reverse solute flux augmented to reach values $J_{\mathrm{w}, 2}$ and $J_{\mathrm{s}, 2}$, respectively, in stage 2 . A third and a fourth stage were then performed in a similar fashion. Values of water flux and reverse solute flux were experimentally measured at every stage. For each stage, addition of solute to the draw solution gives rise to an initial transient state before the solute concentration profiles on both sides of the membrane reach steady state. Allowing the system to attain steady state is necessary before reliable values of water and reverse salt fluxes can be recorded.

\section{Determining the intrinsic transport and structural properties of the membrane}

\subsection{Water and salt flux governing equations in FO}

The mass transport across a membrane in FO can be expressed in terms of the membrane characteristic properties, the hydrodynamics in the membrane flow cell, and experimentally accessible parameters: the bulk solute concentration of the draw solution, $c_{\mathrm{D}}$, feed solution concentration, $c_{\mathrm{F}}$, and the corresponding osmotic pressures, $\pi_{\mathrm{D}}$ and $\pi_{\mathrm{F}}$. The derivation of the FO water and salt flux equations presented in Appendix A follows the approach adopted to derive the governing equations in PRO in our previous publication [35]. It should be noted that salt and draw solute are used interchangeably in the manuscript. The derivation yields the following expressions for the water flux, $J_{\mathrm{w}}$, and the reverse salt flux, $J_{\mathrm{s}}$, in $\mathrm{FO}$

$J_{w}=A\left\{\frac{\pi_{D} \exp \left(-\frac{J_{W} S}{D}\right)-\pi_{F} \exp \left(\frac{J_{W}}{k}\right)}{1+\frac{B}{J_{w}}\left[\exp \left(\frac{J_{w}}{k}\right)-\exp \left(-\frac{J_{W} S}{D}\right)\right]}\right\}$

$J_{S}=B\left\{\frac{c_{D} \exp \left(-\frac{J_{W} S}{D}\right)-c_{F} \exp \left(\frac{J_{w}}{k}\right)}{1+\frac{B}{J_{w}}\left[\exp \left(\frac{J_{w}}{k}\right)-\exp \left(-\frac{J_{w} S}{D}\right)\right]}\right\}$

where $k$ is the feed solute mass transfer coefficient and $D$ is the bulk diffusion coefficient of the draw salt. The water permeability coefficient, $A$, and salt permeability coefficient, $B$, are intrinsic properties of the membrane active layer. The support layer structural parameter, $S$, is defined as $t_{s} \tau / \varepsilon$, with $t_{\mathrm{s}}$ being the thickness of the support layer, $\tau$ its tortuosity, and $\varepsilon$ its porosity. In these equations, the terms $\exp \left(J_{w} / k\right)$ and $\exp \left(-J_{w} S / D\right)$ account for concentrative external concentration polarization (ECP) and dilutive internal concentration polarization (ICP), respectively. The membrane characteristic parameters can be determined numerically by solving a system of equations if all the other variables, water and salt flux, feed channel mass transfer coefficient, salt diffusivity, and concentrations or osmotic pressures of the solutions, are known.

\subsection{Calculating $A, B$, and $S$ numerically by minimization of a global error}

We have developed an algorithm to calculate the membrane parameters, $A, B$, and $S$, from experimental water and salt flux data. By following the four stage procedure outlined in Section 2, eight experimental measurements were collected during a single FO experiment, namely $J_{\mathrm{w}, i}$ and $J_{\mathrm{s}, i}$, where $i=1,2,3,4$ denotes the stage of the experiment (Fig. 1). The concentrations and corresponding osmotic pressures of the draw and feed solutions at each stage were also recorded. The FO transport Eqs. (1) and (2) were fitted to the experimental fluxes by least-squares non-linear fitting, using $A, B$, and $S$ as regression parameters, and $c_{\mathrm{D}}\left(\pi_{\mathrm{D}}\right)$ and $c_{\mathrm{F}}\left(\pi_{\mathrm{F}}\right)$ as independent experimental variables. $D$, the bulk diffusion coefficient of aqueous $\mathrm{NaCl}$, was treated as a known parameter, its value being set to $1.48 \times 10^{-9} \mathrm{~m}^{2} / \mathrm{s}$ [36]. The treatment of $k$, the feed solute mass transfer coefficient, is discussed in the following section.

The eight transport equations and three unknowns ( $A, B$ and $S$ ) constitute an over-determined system of non-linear equations, amenable for numerical solution by least-squares minimization of the global error in the calculated fluxes relative to the experimental values. Specifically, the global error, $E$, is defined as the non-dimensional sum of the offsets in the water and salt fluxes

$E=E_{w}+E_{s}=\sum_{i=1}^{n}\left(\frac{J_{w, i}^{E X P}-J_{w, i}^{C A L C}}{J_{w}^{E X P, n}}\right)^{2}+\sum_{i=1}^{n}\left(\frac{J_{s, i}^{E X P}-J_{s, i}^{C A L C}}{\bar{J}_{s}^{E X P, n}}\right)^{2}$

In this equation, $n$ is the number of stages and had a value of 4 for the protocol described in this study, and the superscripts EXP and CALC indicate experimental and calculated (from Eqs. (1) and (2)) fluxes, respectively. The quantity $\bar{J}_{w}^{E X P, n}$ is the mean experimental water flux over the four FO stages, i.e., $\bar{J}_{w}^{E X P, n}=\left(\Sigma_{i=1}^{n} J_{w, i}^{E X P}\right) / n$. The error at each stage was scaled by $\bar{J}_{w}^{E X P, n}$, so that each term in Eq. (3) is weighed equally, avoiding spurious biasing of the global error due to the different orders of magnitude of $J_{w, i}^{E X P}$. The same scaling was performed for the error relative to the salt fluxes.

Given that the water flux equation is an implicit function of $J_{w, i}^{C A L C}$, minimization of the objective function (Eq. (3)) is subject to closure of Eq. (1). Mathematically, this implies that the minimization must be subjected to the non-linear equality constraint

$J_{w, i}^{C A L C}-A\left\{\frac{\pi_{D, i} \exp \left(-\frac{J_{w, i}^{C A L C} S}{D}\right)-\pi_{F, i}}{1+\frac{B}{J_{w, i}^{C A L C}}\left[1-\exp \left(-\frac{J_{w, i}^{C A L C} S}{D}\right)\right]}\right\}=0$

for $i=1, \ldots, n$. Eq. (4) assumes that the effect of ECP is negligible (i. e., $\exp \left(J_{w} / k\right)=1$ in Eq. (1)). Justification of this assumption is given in Section 3.3.

Three different initial estimates were used to initiate the iterating calculations for $A, B$, and $S$. The first set contained values that were smaller than the expected results, namely $0.1 \mathrm{~L} \mathrm{~m}^{-2} \mathrm{~h}^{-1} \mathrm{bar}^{-1}, 0.01 \mathrm{~L} \mathrm{~m}^{-2} \mathrm{~h}^{-1}$, and $50 \mu \mathrm{m}$ for $A, B$, and $S$, respectively. The third estimates had larger values of $5 \mathrm{~L} \mathrm{~m}^{-2} \mathrm{~h}^{-1}$ bar $^{-1}, 1 \mathrm{~L} \mathrm{~m}^{-2} \mathrm{~h}^{-1}$, and $1000 \mu \mathrm{m}$ for the three parameters. The second set of $1.5 \mathrm{~L} \mathrm{~m}^{-2} \mathrm{~h}^{-1} \mathrm{bar}^{-1}, 0.3 \mathrm{~L} \mathrm{~m}^{-2} \mathrm{~h}^{-1}$, and $300 \mu \mathrm{m}$ consisted of values that were assumed to be closer to the results. The algorithm is implemented to accept the solution associated with the lowest $E$ from the three possible solutions related to each set of initial estimates.

The goodness of the fit was assessed by computing the coefficient of determination, which for the water flux is given by

$R_{w}^{2} \equiv 1-\frac{S S_{e r r, w}}{S S_{T O T, w}}=1-\frac{\sum_{i=1}^{n}\left(J_{w, i}^{E X P}-J_{w, i}^{C A L C}\right)^{2}}{\sum_{i=1}^{n}\left(J_{w, i}^{E X P}-J_{w}^{E X P, n}\right)^{2}}$

where $S S_{\text {err }}$ is the residual sum of squares, $S S_{T O T}$ is the total sum of squares, and $n$ is equal to the number of stages, i.e., $n=4$ for the protocol demonstrated here. The coefficient of determination for the salt flux, $R_{s}^{2}$, was calculated analogously.

The algorithm was implemented in two different proprietary software packages: Microsoft Visual Basic within Microsoft Excel (Microsoft Corporation, Redmond, WA) and Matlab (Mathworks Inc., Natick, MA). The input parameters included the average draw and feed solution concentrations in each stage, system temperature, salt diffusion coefficient in the bulk solution, measured water and salt fluxes, and the initial guesses for the parameters to be 
calculated. Both codes find estimates of $A, B$, and $S$ by minimizing Eq. (3) subject to non-linear constraints (Eq. (4)). The two packages used different methods for the convergence of the numerical solutions: Microsoft Excel adopted the generalized reduced gradient method with forward differencing [37], while Matlab employed the sequential quadratic programming (SQP) method. As shown in the Appendix, both implementations yield identical results. Details on the theory underlying each of the numerical methods may be found in standard numerical methods textbooks [38]. In the Matlab implementation, convergence was reached when the relative change in the value of the fitted parameters between successive iterations was less than a tolerance, set here to $1 \times 10^{-6}$, and the maximum constraint violation was less than $1 \times 10^{-5}$. More stringent tolerance values resulted in identical results. The Excel spreadsheet and the Matlab file are available for download, gratis for non-commercial use, from the Supplementary material of the online version of this paper (http:// dx.doi.org/10.1016/j.memsci.2013.05.023).

\subsection{Experimental and modeling assumptions}

In the derivation of Eqs. (1) and (2), the membrane reflection coefficient, $\sigma$, was assumed to have a value of 1 . That is, the membrane has a dense selective layer that is able to maintain virtually the entire osmotic pressure difference across it (i.e., $\sigma$ approaches unity) [39]. Therefore, the proposed methodology is only valid for tight salt-rejecting membranes.

In FO, the membrane support layer faces the draw solution. Water permeating across the active layer dilutes the draw solution in the support layer, resulting in dilutive ICP, the effect of which is to decrease the net osmotic driving force. ICP is partially mitigated by transport of solute from the draw solution into the membrane support layer. However, solute transport within the support layer occurs almost exclusively by diffusion, process dependent on $D$, given that the support layer acts as an unstirred boundary layer [40]. It is important to note that, while the draw solute diffusivity is dependent on the local salt concentration-and therefore differs across the membrane support layer-the mass transport model from which Eqs. (1) and (2) were derived assumes a constant $D$ within the membrane support layer. This simplification is appropriate for the range of concentrations of the sodium chloride draw solution considered in this work, namely $0.05-2.0 \mathrm{M}$. Over this range, $D_{\mathrm{NaCl}}$ varies by less than $3 \%$, between 1.472 and $1.519 \times 10^{-9} \mathrm{~m}^{2} / \mathrm{s}$ [36].

The osmotic pressure generated by the solute was assumed to be related linearly to the solute concentration via the van't Hoff equation, $\pi=v c R_{g} T$, where $v$ is the number of ionic species each solute molecule dissociates into, $R_{\mathrm{g}}$ is the ideal gas constant, and $T$ is the absolute temperature (Appendix A). Although the sodium chloride draw solution deviates from ideal behavior at high concentrations, this relation was applicable for the procedure outlined here because the solute concentration at the support/ active layer interface was significantly lower than that of the bulk solutions, due to the effects of ICP. For example, a $1.5 \mathrm{M} \mathrm{NaCl}$ draw solution in FO yields a support/active layer interface concentration of $\sim 0.3$ and $\sim 0.6 \mathrm{M}$ for hand-cast TFC membranes and commercial asymmetric cellulose triacetate membranes, respectively [29]. The low solute concentration at the support/active layer interface means that the solution will not depart considerably from ideality.

From this assumption follows a linear relationship between the experimental value of $J_{\mathrm{w}} / J_{\mathrm{s}}$ and the ratio $A / B$, namely $\left(J_{w} / J_{s}\right)=(A / B) v R_{g} T[40]$. Because this ratio only depends on intrinsic membrane active layer characteristics, $A$ and $B$ (in addition to $v$ and $T$ ), it is always constant regardless of the concentrations in the draw and feed solutions. The $J_{\mathrm{w}} / J_{\mathrm{s}}$ factor, termed the reverse flux selectivity, can be regarded as a quality control parameter and its near constancy is a necessary condition for the successful use of the proposed methodology (see Sections 4.3 and 5.4).

Lastly, we assumed the detrimental effect of ECP on the active side to be negligible. This assumption is validated trivially by the negligible feed solution concentration throughout the experimental run (we use DI water as feed solution) and the very small reverse draw salt flux, both of which render ECP ineffectual. In addition, the approximation was justified by the hydrodynamic conditions maintained at the stirred boundary layer on the feed side, where typical values of the feed solution mass transfer coefficient, $k$, far exceed the permeating water flux. Accordingly, this assumption was embedded in the algorithms by imposing $k \rightarrow \infty$, and, therefore, that the exponential term $\exp \left(J_{w} / k\right)$ appearing in Eqs. (1) and (2) had a value of 1. We also assumed that ECP in the draw solution is negligible because it is significantly lower than ICP within the support layer.

\section{Implementation of the proposed methodology}

\subsection{FO membranes}

Both hand-cast TFC and commercial membranes were characterized. Hand-cast TFC FO membranes were fabricated adapting the procedure outlined in our previous publications $[22,29]$. Briefly, polysulfone (PSf, $9 \mathrm{wt} \%, M_{\mathrm{n}}$ : 22,000 Da, Sigma-Aldrich, St. Louis, MO) was dissolved in $\mathrm{N}-\mathrm{N}$-dimethylformamide (DMF, anhydrous, 99.8\%, Sigma-Aldrich) by stirring at room temperature $\left(23^{\circ} \mathrm{C}\right)$ for $6 \mathrm{~h}$ and then stored in a desiccator for at least $15 \mathrm{~h}$ prior to casting. To begin casting the membrane, a commercial polyester non-woven fabric (PET, Grade 3249, Ahlstrom, Helsinki, Finland) was taped on a glass plate and was wet with 1-methyl-2pyrrolidinone (NMP, anhydrous, 99.5\%, Sigma-Aldrich). The PSf solution was then drawn down the PET fabric using a casting knife (Gardco, Pompano Beach, FL) with an adjustable gate height fixed at $375 \mu \mathrm{m}(\sim 15 \mathrm{mils})$. The whole composite was immediately immersed in a precipitation bath containing $3 \mathrm{wt} \%$ DMF in deionized (DI) water at room temperature to initiate non-solvent induced phase separation $[41,42]$. The support membrane remained in the precipitation bath for $10 \mathrm{~min}$ before being transferred to a DI water bath for storage until polyamide (PA) formation. The PA active layer was formed on top of the hand-cast PSf support layers via interfacial polymerization [22,29] between 1,3-phenylenediamine (MPD, >99\%, Sigma-Aldrich) dissolved in DI water at $3.4 \mathrm{wt} \%$ and 1,3,5-benzenetricarbonyl trichloride (TMC, 98\%, Sigma-Aldrich) dissolved in Isopar G (Univar, Redmond, WA) at $0.15 \mathrm{wt} \%$. Following this reaction, the membrane was cured in DI water at $95^{\circ} \mathrm{C}$ for $120 \mathrm{~s}$, rinsed with a $200 \mathrm{ppm} \mathrm{NaOCl}$ aqueous solution for $120 \mathrm{~s}$, then soaked in a $1000 \mathrm{ppm} \mathrm{NaHSO}_{3}$ aqueous solution for $30 \mathrm{~s}$, before a final wet curing step at $95^{\circ} \mathrm{C}$ for $120 \mathrm{~s}$. After fabrication, the TFC membranes were rinsed thoroughly and stored in DI water at $4^{\circ} \mathrm{C}$.

Commercial asymmetric cellulose triacetate membranes were acquired from Hydration Technology Innovations (Albany, OR) (HTI-CTA, batch \# 110610-ES-3). Thin-film composite seawater reverse osmosis membranes (SW30, Dow Chemical Company, Midland, MI) were also acquired and deployed. The PET fabric layer on the support layer surface of these samples was removed according to procedures described in our previous study [43]; these membrane samples are designated as "SW30 No PET". Additionally, prototype thin-film composite FO membranes were obtained from Oasys Water (Oasys Water Inc., Boston, MA). All membranes were thoroughly wet prior to the experiments by immersing in $25 \%$ isopropanol solutions for $30 \mathrm{~min}$. Three separate samples for each membrane type were employed. 
A

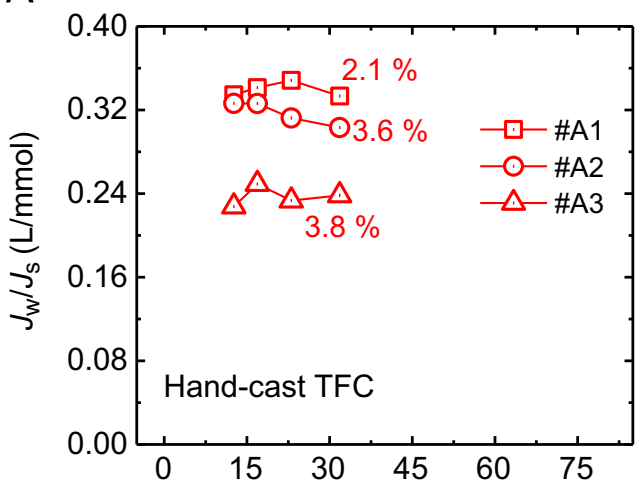

C

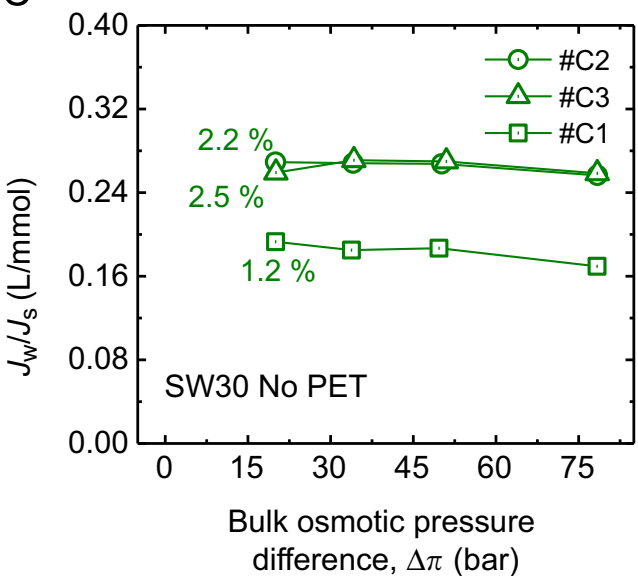

$\mathrm{B}$

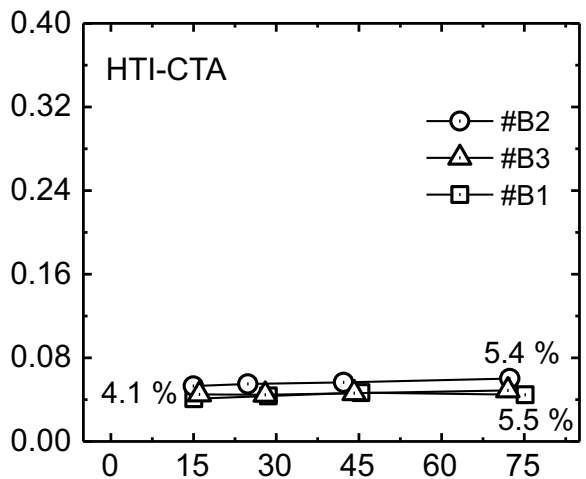

D

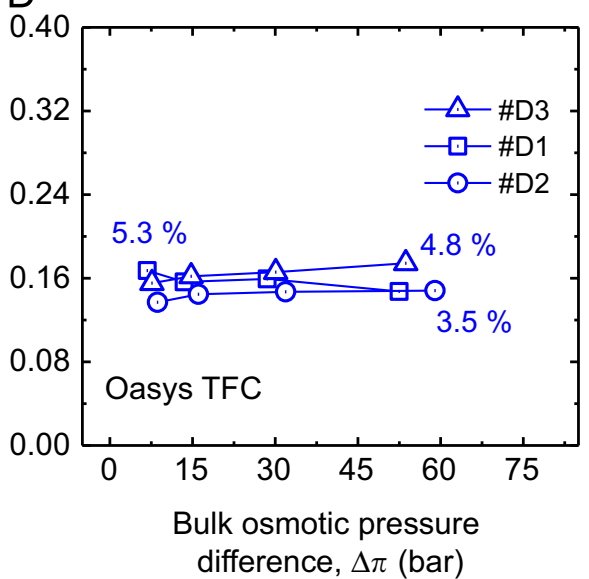

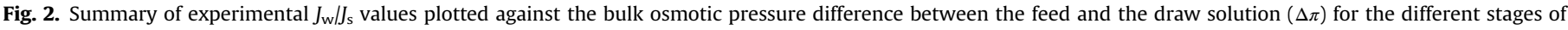

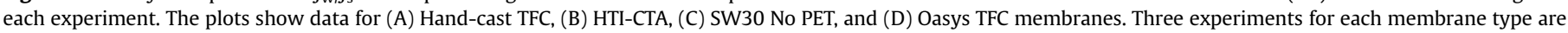

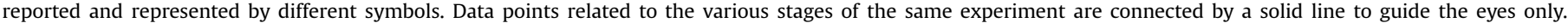
Calculated values of the coefficient of variation (\%) of the $J_{\mathrm{w}} / J_{\mathrm{s}}$ values between the different stages of each experiment are also reported next to the related symbols.

\subsection{FO setup and experimental conditions}

FO water fluxes and reverse solute fluxes were determined in an experimental cross-flow FO system described in our previous studies $[22,35]$. The custom-built cell had an effective membrane area of $20.02 \mathrm{~cm}^{2}$ on both sides of the membrane. The unit was operated with co-current cross-flow without mesh spacers, and variable speed gear pumps (Cole-Parmer, Vernon Hills, IL) were used to circulate the solutions in closed loops at a cross-flow velocity of $17.1 \mathrm{~cm} / \mathrm{s}$. A water bath (Neslab, Newington, $\mathrm{NH}$ ) kept the temperature of both feed and draw solutions constant at $25 \pm 0.5^{\circ} \mathrm{C}$. All characterization tests were conducted with the membrane in FO configuration, i.e., porous support layer facing the draw solution and active layer facing the feed solution. For FO characterization tests, a stock $5 \mathrm{M} \mathrm{NaCl}$ solution was prepared with sodium chloride $(\mathrm{NaCl})$ from J.T. Baker (Phillipsburg, NJ), by dissolving the appropriate amount of $\mathrm{NaCl}$ in DI water (Milli-Q, Millipore, Billerica, MA).

In this study, $\mathrm{NaCl}$ was chosen as draw solute, because it fulfills a number of critical criteria. Specifically, $\mathrm{NaCl}$ (i) is rejected to a large extent ( $>95 \%$ ) by the membrane, (ii) is able to generate a high osmotic pressure, (iii) does not deviate significantly from the van't Hoff equation, (iv) has nearly constant $D$ over the range of concentrations employed, and ( $v$ ) is easily quantifiable in the feed by conductivity measurements to determine $J_{s}$.

In the beginning of the experiment, DI water was circulated on both the draw and feed side to equilibrate the system temperature. An appropriate volume of the $\mathrm{NaCl}$ stock solution was added to the draw solution to obtain the desired concentration and initiate the first stage. After attainment of steady state, the water flux, $J_{w, i}^{E X P}$, was determined by monitoring the rate of change in weight of the draw solution, and the solute concentration in the feed was measured at $1 \mathrm{~min}$ intervals with a calibrated conductivity meter (Oakton Instruments, Vernon Hills, IL). Once the water flux had stabilized, feed concentration and water flux data were logged for at least $15 \mathrm{~min}$. Appropriate amounts of the $\mathrm{NaCl}$ stock solution were then dosed into the draw solution to commence the second stage and data collection was performed again. The procedure was repeated for the third and fourth stages. Tailored concentrations of $\mathrm{NaCl}$ draw solutions were chosen for different types of membranes since transport properties varied. Concentrations were selected to obtain a significant range in the magnitude of water and solute fluxes throughout the stages, while avoiding overly low or high flux values. An appropriate range of fluxes is necessary to ensure instrumental sensitivity during measurement and the validity of the assumptions stated in Section 3.3. The salt concentrations of the draw and feed solutions for all the characterization experiments are presented in Table B1 of the Appendix.

$\mathrm{NaCl}$ reverse flux during stage $i, J_{s, i}^{E X P}$, was calculated from the measured change of concentration in the feed, $c_{\mathrm{F}, \mathrm{i}}$. An $\mathrm{NaCl}$ mole balance in the feed solution yields

$J_{s, i}=\frac{c_{F, i}\left(V_{F 0, i}-J_{w, i} A_{m} t\right)-c_{F 0, i} V_{F 0, i}}{A_{m} t}$

where $c_{F, i}$ is the feed solute concentration, $V_{F O}$ is the initial volume of the feed solution, $A_{m}$ is the membrane area, $t$ is the time elapsed, and $c_{F O}$ is the initial $\mathrm{NaCl}$ concentration. Due to both the permeation of water and the salt leakage, the concentration of the 
draw solution was diluted throughout the experiment. We used suitably large volumes of draw and feed solution to avoid large changes in osmotic pressure within each stage. The concentrations and the volumes of both the draw and the feed solutions were accurately monitored over the entire duration of the experiment. The decrease in draw solution concentration between the start and end of each stage, due to effect of water flux and reverse salt flux, was less than $0.5 \%$.

Membranes were also tested in a reverse osmosis (RO) setup according to the procedure described in our previous publications $[22,29]$. The following conditions were employed: overnight compaction with DI water at 31.1 bar ( $450 \mathrm{psi}$ ) followed by measurements of water flux and salt rejection at applied pressure of $27.6 \mathrm{bar}$ ( $400 \mathrm{psi}$ ), $50 \mathrm{mM} \mathrm{NaCl}$ feed solution, cross-flow of $21.4 \mathrm{~cm} / \mathrm{s}$, and temperature of $25^{\circ} \mathrm{C}$.

\subsection{Experimental results}

The complete set of water and salt fluxes measured during the experiments, together with the draw and feed solution concentrations and osmotic pressures, are tabulated in Table B1 of the Appendix. Fig. 2 presents the experimental results plotted as the $J_{\mathrm{w}} / J_{\mathrm{s}}$ values against the bulk osmotic pressure difference between the feed and the draw side. The latter was calculated from $\mathrm{NaCl}$ concentrations using the van't Hoff equation. The percent figure beside each data series represents the coefficient of variation (CV) calculated between the $J_{\mathrm{w}} / J_{\mathrm{s}}$ values of the different stages in each experiment. Different draw solute concentrations were employed for the various membrane types, due to their dissimilar performance. The experimental results show that $J_{\mathrm{w}} / J_{\mathrm{s}}$ remains approximately constant throughout the stages of each experiment, as expected from theory [40]. In all experiments, the CV was found to be below $6 \%$.

\subsection{Calculated parameters}

Table 1 summarizes the transport parameters and the related coefficients of determination, calculated by the model for each experiment. Only the results obtained using the Excel-based algorithm are presented for conciseness. However, the Matlabbased algorithm produced practically identical results, which are reported in Table $\mathrm{C} 1$ of the Appendix. The largest deviation between the same parameter calculated by the two algorithms was found to be $0.66 \%$, with the majority of values within $0.33 \%$. A noteworthy result was the ability of the algorithms to reach a univocal solution regardless of the initial estimates for $A, B$, and $S$ and notwithstanding the different minimization algorithms (results not shown in manuscript). In addition, all the simulations yielded values of the coefficient of determination larger than 0.95 for both water and salt fluxes. In most cases, the $R^{2}$ values were larger than 0.99 .

The projected water and salt fluxes of the membranes, based on the characteristic parameters, $A, B$, and $S$, determined from the regression, can be numerically calculated using Eqs. (1) and (2). The values of the measured and calculated water and salt fluxes are compared on a linear-linear plot in Fig. 3 (solid symbols). The agreement between the model and experiments was good, as demonstrated by the clustering of data near the dashed line (slope $=1$ ), which represents perfect agreement between experimental data and model calculations. The equivalence among the two separate algorithms, the robustness of the calculation with respect to the initial parameter estimates, and the high coefficients of determination suggest that the methodology is reliable and the calculation codes well posed. Finally, we note how the calculated values of $A, B$, and $S$, were similar for the different coupons of each membrane type.

\section{Robustness and reliability of the methodology}

\subsection{Comparison to current approach}

It is important to make a comparison between the parameters calculated using our method and those obtained by the current approach, whereby membrane active layer properties are measured in an RO experiment and the structural parameter is calculated using additional FO measurements. In this study, RO experiments were performed on the same membrane coupons after FO characterization to determine $A_{R O}$ and $B_{R O}$ from water flux and salt rejection readings. These parameters were then applied to Eq. (1) in combination with the water fluxes measured in the FO experiments described earlier in the paper (Sections 2 and 4.2) in order to calculate the membrane structural parameter, $S_{R O+F O}$.

The values of $A_{R O}, B_{R O}$, and $S_{R O+F O}$ are summarized in Table 2 alongside the corresponding values determined using our FO protocol. The values of $S_{R O+F O}$ presented in Table 2 are the average results obtained by applying $A_{R O}$ and $B_{R O}$ in the four stages of each respective FO experiment. Agreement between the active layer properties determined by the two methods was generally poor. The average discrepancy is approximately $46 \%$, with the largest deviation observed in the membrane salt permeabilities. This difference in the active layer parameters inevitably translates to deviations between the structural parameters obtained in the two configurations. However, the calculated parameters from the $\mathrm{RO} \rightarrow$ FO methodology have relatively low standard deviations,

Table 1

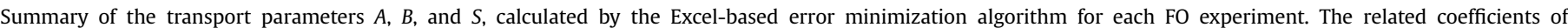

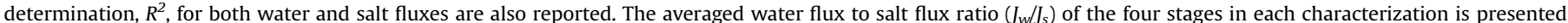
along with the corresponding coefficient of variation (CV).

\begin{tabular}{|c|c|c|c|c|c|c|c|c|}
\hline Membrane type & Sample & $\boldsymbol{A}\left(\mathrm{L} \mathrm{m}^{-2} \mathrm{~h}^{-1} \mathrm{bar}^{-1}\right)$ & $\boldsymbol{B}\left(\mathrm{L} \mathrm{m}^{-2} \mathrm{~h}^{-1}\right)$ & $\boldsymbol{S}(\mu \mathrm{m})$ & $R^{2}\left[J_{w}\right]$ & $R^{2}\left[J_{s}\right]$ & Average $J_{\mathrm{w}} / J_{\mathbf{s}}(\mathrm{L} / \mathrm{mmol})$ & $\mathbf{C V}(\%)$ \\
\hline \multirow[t]{3}{*}{ Hand-cast TFC } & \#A1 & 1.61 & 0.24 & 400 & 0.998 & 0.996 & 0.339 & 2.05 \\
\hline & \#A2 & 1.23 & 0.20 & 328 & 0.994 & 0.990 & 0.317 & 3.57 \\
\hline & \#A3 & 1.21 & 0.25 & 395 & 0.992 & 0.997 & 0.237 & 3.84 \\
\hline \multirow[t]{3}{*}{ HTI-CTA } & \#B1 & 1.22 & 1.35 & 481 & 0.997 & 0.976 & 0.044 & 5.52 \\
\hline & \#B2 & 1.17 & 1.00 & 473 & 0.994 & 0.992 & 0.056 & 5.44 \\
\hline & \#B3 & 1.63 & 1.73 & 541 & 0.989 & 0.993 & 0.046 & 4.10 \\
\hline \multirow[t]{3}{*}{ SW30 No PET } & \#C1 & 1.32 & 0.36 & 1620 & 0.963 & 0.982 & 0.184 & 5.43 \\
\hline & $\# C 2$ & 1.05 & 0.20 & 1540 & 0.994 & 0.983 & 0.265 & 2.23 \\
\hline & \#C3 & 1.27 & 0.24 & 1510 & 0.996 & 0.994 & 0.265 & 2.54 \\
\hline \multirow[t]{3}{*}{ Oasys TFC } & \#D1 & 3.69 & 1.19 & 424 & 0.992 & 0.989 & 0.158 & 5.28 \\
\hline & \#D2 & 3.37 & 1.14 & 589 & 0.998 & 0.997 & 0.144 & 3.48 \\
\hline & \#D3 & 4.26 & 1.26 & 526 & 0.996 & 0.993 & 0.164 & 4.84 \\
\hline
\end{tabular}


A

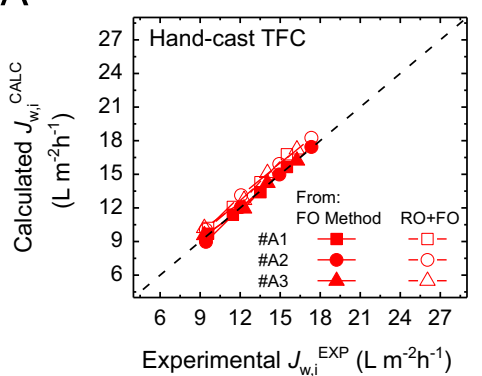

E

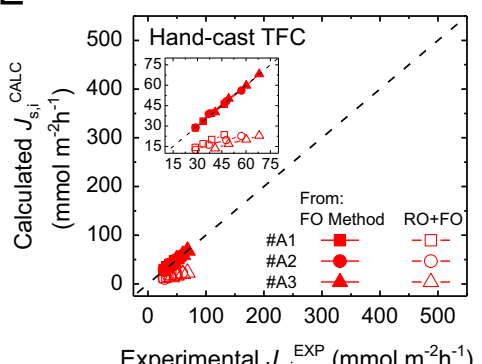

B

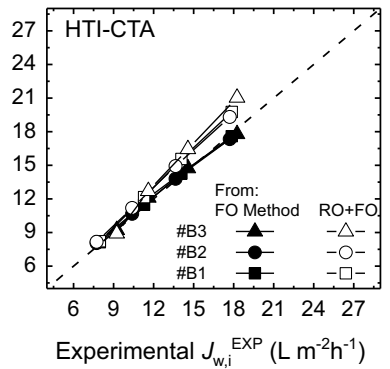

$\mathrm{F}$

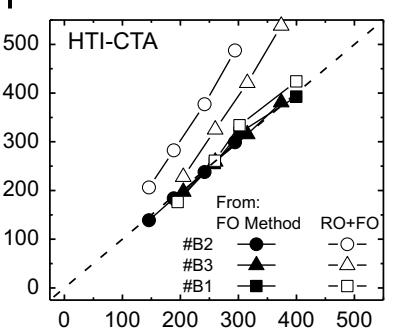

Experimental $J_{\mathrm{s}, \mathrm{i}}^{\mathrm{EXP}}\left(\mathrm{mmol} \mathrm{m} \mathrm{m}^{-2} \mathrm{~h}^{-1}\right)$
C

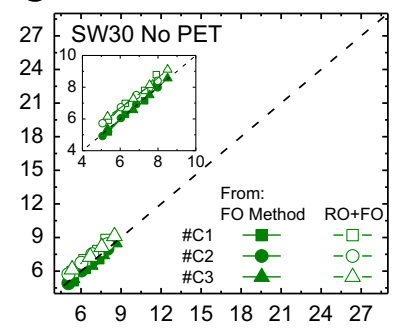

Experimental $J_{w, i} \operatorname{EXP}\left(\mathrm{L} \mathrm{m}^{-2} \mathrm{~h}^{-1}\right)$

G

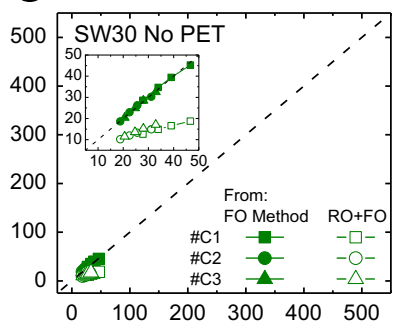

Experimental $J_{\mathrm{s}, \mathrm{i}} \operatorname{EXP}\left(\mathrm{mmol} \mathrm{m} \mathrm{m}^{-2} \mathrm{~h}^{-1}\right)$
D

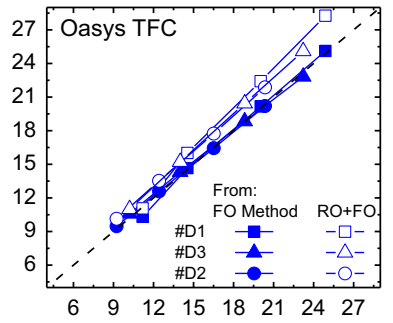

Experimental $J_{w, i}{ }^{\operatorname{EXP}}\left(\mathrm{L} \mathrm{m}^{-2} \mathrm{~h}^{-1}\right)$

\section{$\mathrm{H}$}

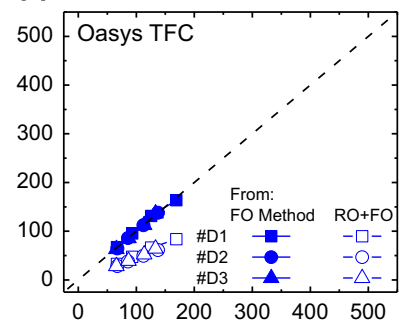

Experimental $\left.J_{\mathrm{s}, \mathrm{i}} \mathrm{EXP}^{\mathrm{mmol} \mathrm{m}} \mathrm{m}^{-2} \mathrm{~h}^{-1}\right)$

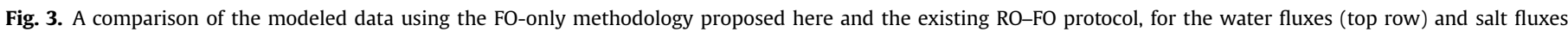

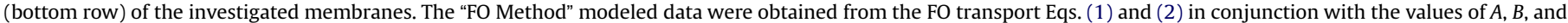

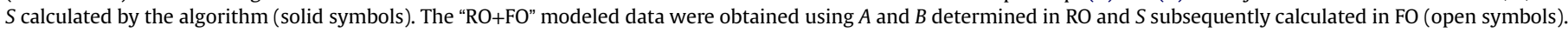

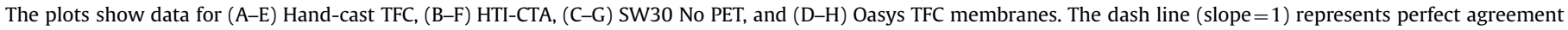
between experimental data and calculations.

\section{Table 2}

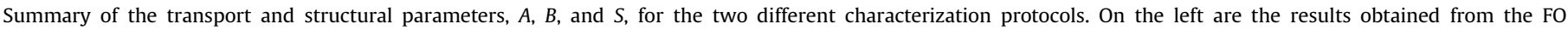

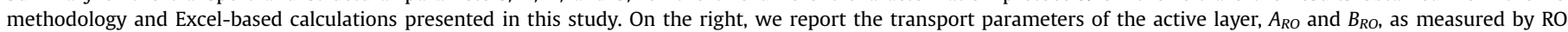

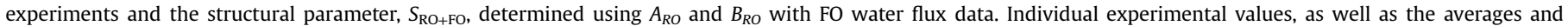
standard deviations, for each membrane type are presented.

\begin{tabular}{|c|c|c|c|c|c|c|c|c|c|c|}
\hline \multirow[t]{2}{*}{ Membrane type } & \multirow[t]{2}{*}{ Sample } & \multicolumn{4}{|l|}{ FO method } & \multicolumn{4}{|l|}{ RO+FO } & \multirow[b]{2}{*}{$\begin{array}{l}\frac{(A / B)_{F O}}{(A / B)_{R O}} \\
(\%)\end{array}$} \\
\hline & & $\begin{array}{l}A \\
\left(\mathrm{~L} \mathrm{~m}^{-2} \mathrm{~h}^{-1} \mathrm{bar}^{-1}\right)\end{array}$ & $\begin{array}{l}\boldsymbol{B} \\
\left(\mathrm{L} \mathrm{m}^{-2} \mathrm{~h}^{-1}\right)\end{array}$ & $\begin{array}{l}\boldsymbol{S} \\
(\mu \mathrm{m})\end{array}$ & $\begin{array}{l}(\boldsymbol{A} / \mathbf{B})_{\mathbf{F O}} \\
\left(\operatorname{bar}^{-1}\right)\end{array}$ & $\begin{array}{l}A_{\mathbf{R O}} \\
\left(\mathrm{L} \mathrm{m}^{-2} \mathrm{~h}^{-1} \mathrm{bar}^{-1}\right)\end{array}$ & $\begin{array}{l}\boldsymbol{B}_{\mathbf{R O}} \\
\left(\mathrm{L} \mathrm{m}^{-2} \mathrm{~h}^{-1}\right)\end{array}$ & $\begin{array}{l}\boldsymbol{S}_{\mathbf{R O}+\mathbf{F O}} \\
(\mu \mathrm{m})\end{array}$ & $\begin{array}{l}(\boldsymbol{A} / \boldsymbol{B})_{\mathbf{R O}} \\
\left(\mathrm{bar}^{-1}\right)\end{array}$ & \\
\hline \multirow[t]{4}{*}{ Hand-cast TFC } & \#A1 & 1.61 & 0.24 & 400 & 6.85 & 1.58 & 0.11 & 347 & 14.38 & 47.6 \\
\hline & \#A2 & 1.23 & 0.20 & 328 & 6.28 & 1.63 & 0.10 & 383 & 16.28 & 38.6 \\
\hline & \#A3 & 1.21 & 0.25 & 395 & 4.80 & 1.30 & 0.09 & 379 & 15.14 & 31.7 \\
\hline & Avg. & $1.35 \pm 0.23$ & $0.23 \pm 0.03$ & $374 \pm 40$ & $5.98 \pm 1.06$ & $1.50 \pm 0.18$ & $0.10 \pm 0.01$ & $370 \pm 20$ & $15.27 \pm 0.6$ & $39.3 \pm 8.0$ \\
\hline \multirow[t]{4}{*}{ HTI-CTA } & \#B1 & 1.22 & 1.35 & 481 & 0.90 & 0.97 & 1.04 & 342 & 0.94 & 96.1 \\
\hline & \#B2 & 1.17 & 1.00 & 473 & 1.17 & 0.99 & 1.24 & 349 & 0.80 & 146.4 \\
\hline & \#B3 & 1.63 & 1.73 & 541 & 0.94 & 0.94 & 1.19 & 286 & 0.79 & 119.5 \\
\hline & Avg. & $1.34 \pm 0.25$ & $1.36 \pm 0.37$ & $498 \pm 37$ & $1.01 \pm 0.14$ & $0.97 \pm 0.03$ & $1.16 \pm 0.11$ & $326 \pm 35$ & $0.84 \pm 0.08$ & $120.7 \pm 25.2$ \\
\hline \multirow[t]{4}{*}{ SW30 No PET } & \#C1 & 1.32 & 0.36 & 1620 & 3.66 & 2.31 & 0.24 & 1820 & 9.47 & 38.6 \\
\hline & \#C2 & 1.05 & 0.20 & 1540 & 5.33 & 2.49 & 0.22 & 1980 & 11.38 & 46.9 \\
\hline & \#C3 & 1.27 & 0.24 & 1510 & 5.31 & 2.38 & 0.22 & 1750 & 10.77 & 49.3 \\
\hline & Avg. & $1.21 \pm 0.14$ & $0.27 \pm 0.09$ & $1560 \pm 57$ & $4.77 \pm 0.96$ & $2.39 \pm 0.09$ & $0.23 \pm 0.01$ & $1850 \pm 117$ & $10.54 \pm 0.97$ & $44.9 \pm 5.6$ \\
\hline \multirow[t]{4}{*}{ Oasys TFC } & \#D1 & 3.69 & 1.19 & 424 & 3.10 & 3.43 & 0.50 & 346 & 6.84 & 45.4 \\
\hline & \#D2 & 3.37 & 1.14 & 589 & 2.96 & 3.30 & 0.46 & 527 & 7.22 & 41.0 \\
\hline & \#D3 & 4.26 & 1.26 & 526 & 3.38 & 3.58 & 0.45 & 428 & 7.89 & 42.8 \\
\hline & Avg. & $3.77 \pm 0.45$ & $1.20 \pm 0.06$ & $513 \pm 83$ & $3.15 \pm 0.22$ & $3.44 \pm 0.14$ & $0.47 \pm 0.03$ & $434 \pm 91$ & $7.31 \pm 0.53$ & $43.1 \pm 2.2$ \\
\hline
\end{tabular}

indicating that, similar to the FO protocol, it is capable of obtaining consistent and distinct results.

Using the calculated $A_{R O}, B_{R O}$, and $S_{R O+F O}$ values, the water and salt fluxes were determined with governing Eqs. (1) and (2), and plotted in Fig. 3 (open symbols) to compare between the measured and predicted fluxes. Overall, membrane parameters derived using the $\mathrm{RO} \rightarrow \mathrm{FO}$ method gave poorer prediction of the water and salt fluxes, compared to the FO-only approach proposed here (solid symbols). This is evident from the fluxes calculated with $A_{R O}, B_{R O}$, and $S_{R O+F O}$ falling further away from the $45^{\circ}$ dashed line representing perfect agreement between experimental data and model calculations. The deviation of the calculated values was especially pronounced for the reverse salt fluxes, where the higher reverse flux selectivity $(A / B)$ determined for TFC membranes using the $\mathrm{RO} \rightarrow \mathrm{FO}$ method (Table 2 ) resulted in predicted salt fluxes that are significantly lower than the experimental FO values (Fig. 3(E), $(\mathrm{G})$, and $(\mathrm{H})$ ).

The accuracy of the membrane parameters obtained by the two methods can be more clearly contrasted by using governing Eqs. (1) and (2) with the parameter values in Table 2 to predict fluxes as a function of the bulk osmotic pressure difference for Hand-cast TFC \#A1 and Oasys TFC \#D1 (Fig. 4(A) and (B), 


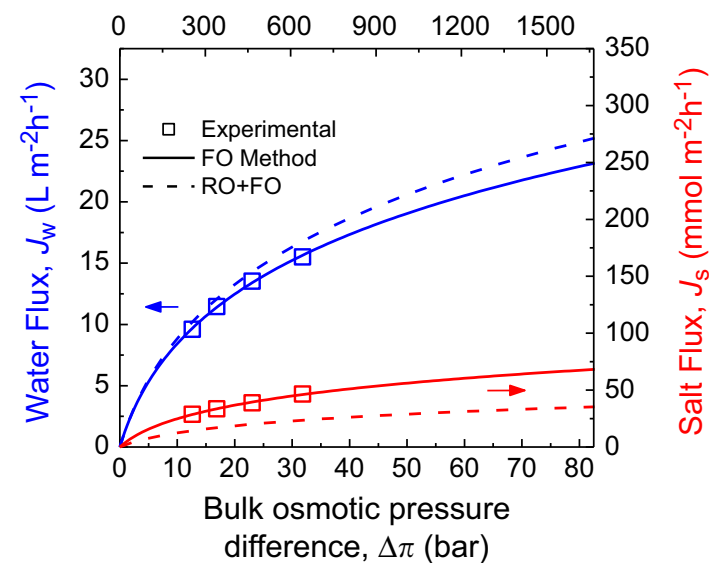

B

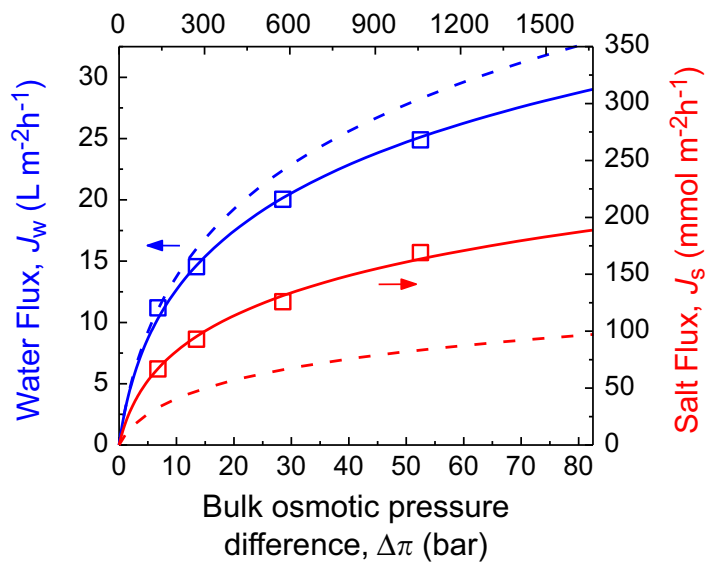

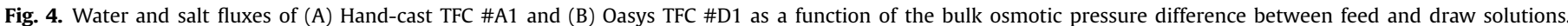

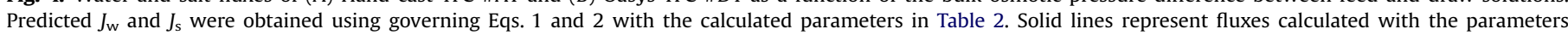

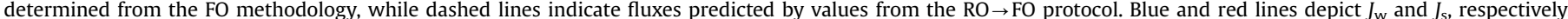

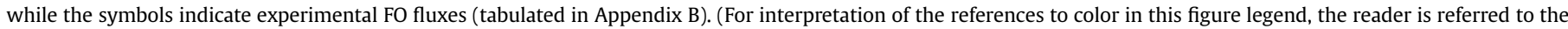
web version of this article.)

respectively). The solid lines represent the fluxes calculated with the parameters determined from the FO methodology, while dashed lines indicate fluxes predicted by $A, B$, and $S$ values from the $\mathrm{RO} \rightarrow \mathrm{FO}$ protocol. Blue and red lines depict $J_{\mathrm{w}}$ and $J_{s}$, respectively, while the symbols indicate experimental FO fluxes. For both membranes, the $\mathrm{RO} \rightarrow \mathrm{FO}$ calculated parameters over-predicted water fluxes (solid lines) and grossly under-predicted reverse salt fluxes (dashed lines). On the other hand, membrane parameters determined by the FO protocol yielded fluxes that are in close agreement with experimental data. These discrepancies highlight the inadequacy of the conventional method to accurately describe and predict the water flux performance and reverse draw salt leakage of membranes in forward osmosis processes.

\subsection{The observed discrepancy requires further analysis}

A noteworthy trend emerged upon examining the calculated $A / B$ ratios, a parameter quantifying the permeability-selectivity of the membrane active layer (Table 2). For the three TFC membranes tested (Hand-cast, Oasys, and SW30), A/B determined using the FO protocol was always lower (31-49\%) than $A / B$ determined in RO. The asymmetric HTI-CTA membrane, however, did not follow the same tendency. Instead, we saw an opposite behavior where the $A / B$ ratios in FO are slightly higher than those in RO. The distinct and consistent trend observed for all three polyamide TFC membranes, but not the integrally skinned cellulose triacetate membrane, coupled with the relative consistency of the calculated parameters with both characterization methods, strongly suggests that this discrepancy is not coincidental or due to erroneous calculations in either methods, but may instead be due to changes in active layer properties or transport mechanism within this layer between RO and FO.

A detailed discussion on the cause of the discrepancy between the parameters measured by our FO methodology and those obtained in $\mathrm{RO} \rightarrow \mathrm{FO}$ experiments is beyond the scope of this study. Nonetheless, we postulate that the observed discrepancies might be related to the difference in driving force between FO, which is driven by an osmotic gradient, and RO, where an applied hydraulic pressure drives mass transfer. Compaction in RO due to applied hydraulic pressure has been shown to increase the $A / B$ ratio in several types of TFC membranes [44]. A recent study found the salt flux of two types of TFC membranes decreased under hydraulic pressure while the water flux remained relatively constant [34]. Under the same conditions, no significant changes were observed with an integrally skinned cellulose triacetate membrane. Other work has also reported disagreement between recorded values of the $A / B$ ratio (or the equivalent $J_{w} / J_{s}$ ratio) obtained in FO and in RO experiments $[45,46]$. The difference in membrane performance reported in past studies suggests that the driving force has certain influence on mass transport across the membrane active layer, and plausibly explains the discrepancies observed here. However, this is only one hypothesis and further investigations on the phenomenon are necessary.

\subsection{Influence of the number of stages}

The least-squares non-linear regression method presented here utilizes an over-determined system of equations to calculate the membrane properties in FO. Hence, to determine the three characteristic parameters, $A, B$, and $S$, four or more flux measurements are needed. That is, the shortest possible experiment would involve two stages to record two pairs of water and reverse salt fluxes. Performing the membrane characterization over a greater number of stages would enhance the accuracy of the results, as inevitable experimental errors are averaged out by a larger dataset. However, experiment brevity is sacrificed when doing so. Here, we carry out a systematic analysis of the influence of the number of stages on the goodness of fit and accuracy of membrane parameters calculated by the proposed method.

Full details of the analytical approach are presented in Appendix D. Briefly, hypothetical characterization experiments were performed on a model membrane of known properties (i.e., water and salt permeability, and structural parameter). To simulate uncertainty in experimental measurements, errors ranging from 0 to $15 \%$ were deliberately introduced to the water and reverse salt flux readings. The simulated fluxes (offset by random errors), along with the corresponding draw and feed solution osmotic pressures and concentrations, were input into the Excel algorithm described in Section 3 to determine the membrane parameters $A, B$, and $S$ that best fit the simulated $J_{\mathrm{w}}$ and $J_{\mathrm{s}}$ "measurements". The deviation of the calculated parameters from the true membrane values represents an assessment of the robustness of the method to unavoidable random experimental errors. 
A

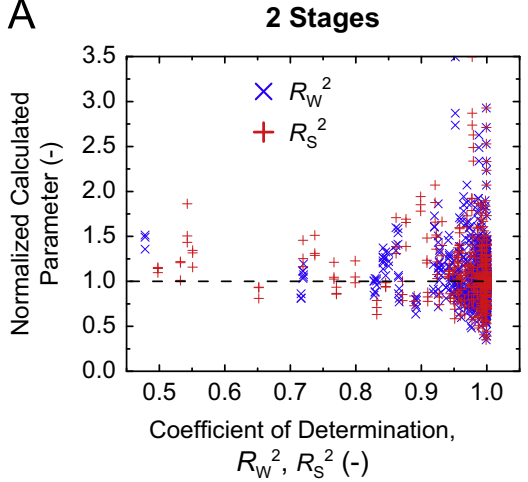

D

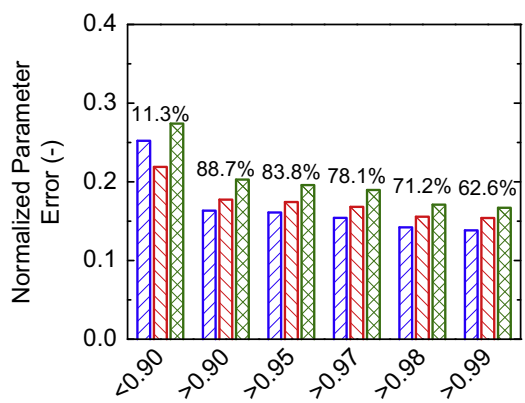

Coefficients of Determination

$$
R_{\mathrm{W}}{ }^{2} \& R_{\mathrm{S}}{ }^{2}(-)
$$

B

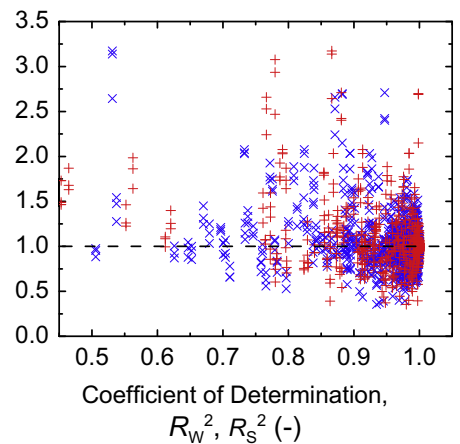

E

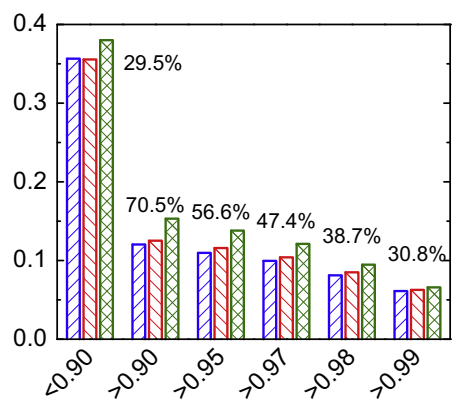

Coefficients of Determination, $R_{\mathrm{W}}{ }^{2} \& R_{\mathrm{S}}{ }^{2}(-)$

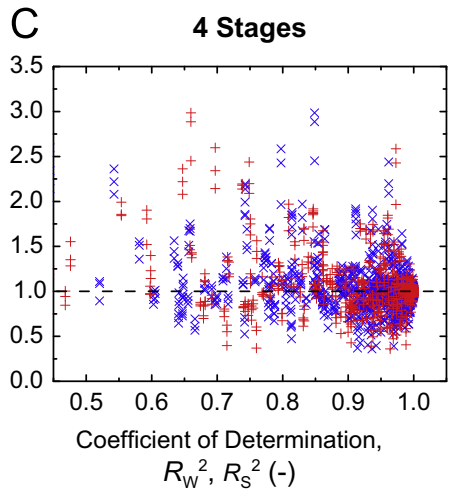

F

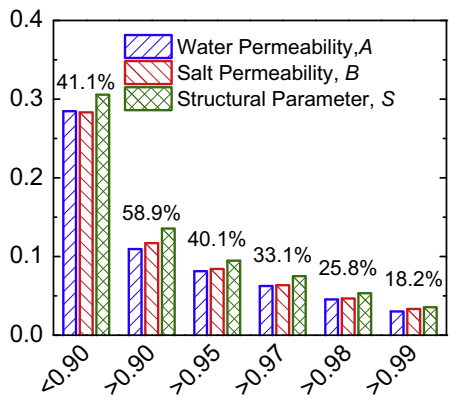

Coefficients of Determination, $R_{\mathrm{W}}^{2} \& R_{\mathrm{S}}^{2}(-)$

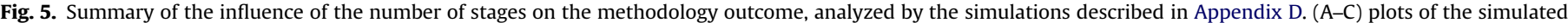

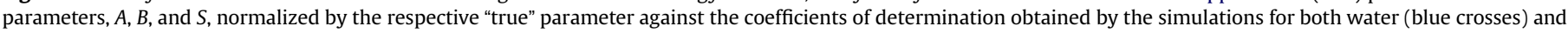

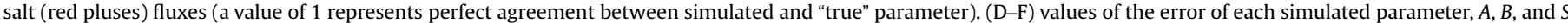

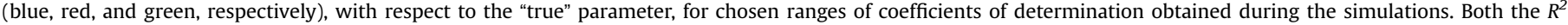

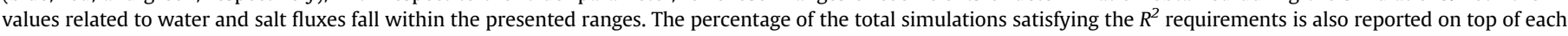

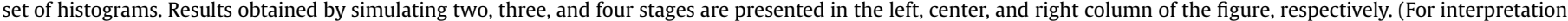
of the references to color in this figure legend, the reader is referred to the web version of this article.)

In actual experiments, the extent of imprecision in the flux readings would be blind to the investigator. Instead, the coefficients of determination (Eq. (5)), an indicator of the goodness of fit, are the most readily available alternative to gauge data quality. The calculated membrane properties of the simulated characterization experiments were normalized (i.e., divided by the true value) and presented in Fig. 5(A-C) as a function of $R^{2}$ for both water and reverse salt flux (blue cross and red plus symbols, respectively). That is, each data point represents the calculated $A$, $B$, or $S$ and the corresponding $R_{\mathrm{w}}{ }^{2}$ and $R_{\mathrm{S}}{ }^{2}$ from the regression. Fig. 5(A)-(C) shows the results of the characterizations carried out in two, three, and four stages, respectively. As the number of stages in the characterization protocol increases, two trends were noted. First, the symbols gradually spread to the left (i.e., lower coefficients of determination) to indicate relatively poorer fits and, second, the data points cluster towards the normalized value of one (horizontal dashed line). Both these observations were anticipated. The coefficients of determination were lower as good fittings are progressively more difficult with the same magnitude of error in a greater number of flux measurements. But with more stages, the intentionally induced experimental errors were averaged out by the greater sample size (from four $J_{\mathrm{w}}$ and $J_{\mathrm{s}}$ values in the two-stage experiments to eight measurements when characterizations were performed in four stages), leading to the calculated parameters to be closer to the true membrane properties.

To evaluate the suitability of the coefficient of determination as a criterion to screen for poor membrane property predictions due to substandard flux data, the normalized error of the calculated parameters (defined as Iparameter $r_{\text {true }}$ - parameter $_{\text {simulated }}$ l/ parameter $r_{\text {true }}$ ) were plotted as a function of the coefficients of determination, for simulated characterizations carried out in two, three, and four stages (Fig. 5(D)-(F), respectively). The horizontal axis in Fig. 5(D-F) denotes the cutoff range for the coefficients of determination of both water and salt fluxes. The percentage indicated above each group of membrane parameters (water permeability, salt permeability, and structural parameter designated by blue, red, and green patterned bars, respectively) represents the fraction of the entire sample with both $R_{\mathrm{w}}{ }^{2}$ and $R_{\mathrm{s}}{ }^{2}$ greater than the cutoff value indicated in the horizontal axis, while the normalized error presented is the average of the each filtered sample subset. For instance, in characterization experiments with three stages, defining the benchmark as both $R_{\mathrm{w}}{ }^{2}$ and $R_{\mathrm{s}}{ }^{2}$ having to be greater than 0.95 results in average normalized error of $11.0 \%$, $11.6 \%$, and $13.8 \%$ for $A, B$, and $S$, respectively (Fig. $5(\mathrm{E})$ ).

Regardless of the number of stages, the magnitudes of error are very similar for water permeability, salt permeability, and structural parameter, indicating the characterization protocol is not lopsidedly biased towards any of the membrane properties in the calculations. Within each characterization, the error in the calculated parameter decreases as the coefficient of determination requirement is raised, while the portion of sample population meeting the cutoff drops. Thus, setting a stricter requirement for the coefficients of determination, an indicator of water and reverse salt flux measurement quality, eliminates the weaker data and minimizes the likelihood of the calculated properties deviating exceedingly from the actual values. As the number of stages increases, the calculated membrane parameters fall closer to the true values for the same coefficient of determination benchmark, 
but the fraction of the sample population fulfilling the criteria is also reduced. That is, by increasing the number of stages, good fits (high $R^{2}$ ) are achieved only with flux measurements with small experimental errors, but the parameter predictions are more likely to be closer to the actual value of the membrane. Note that characterizations performed in two stages yielded relatively poor accuracies, even when the strictest constraint was imposed (Fig. 5 (D), $\sim 15 \%$ error for both $R_{\mathrm{w}}{ }^{2}$ and $R_{\mathrm{s}}{ }^{2}>0.99$ ). This reveals the inherent inability of a two-stage experiment, where just four flux measurements are used to determine the three membrane parameters, to cope with experimental uncertainties.

The actual characterizations of the hand-cast TFC membranes and the commercial HTI-CTA, SW30 No PET, and Oasys TFC membranes, as discussed in Section 4.4 and presented in Table 1, yielded coefficients of determination between 0.963 and 0.998 , with a mean of 0.991 . Based on the above analysis, the excellent fitting of the predicted fluxes to the experimental water and salt flux measurements signifies the calculated membrane parameters are, on average, less than 3.5\% from their true values (Fig. 5(F)). This reinforces the reliability of the proposed methodology to determine the intrinsic transport and structural properties of forward osmosis membranes accurately. A reasonable deviation of $\sim 12 \%$ from the true membrane values was achieved by having both $R_{\mathrm{w}}{ }^{2}$ and $R_{\mathrm{s}}{ }^{2}$ greater than 0.95 in the 3-stage characterization (Fig. 5(E)). This suggests that a briefer experiment with three stages may be adequate to determine the membrane properties if the exactness of the calculated parameters is not a high priority.

\subsection{Influence of the consistency in experimental $J_{w} / J_{s}$ throughout the stages}

The reverse flux selectivity, the ratio of water flux to reverse salt flux, is a constant factor that is solely dependent on the intrinsic membrane active layer characteristics, $A$ and $B$, for the solution concentrations used in this study (Section 3.3). Hence, fluctuations in the $J_{\mathrm{w}} / J_{\mathrm{s}}$ ratio between the stages of the characterization protocol can serve as another indicator for data quality. The calculated membrane parameters are normalized by the true values and plotted in Fig. 6 (A) as a function of the coefficient of variation of $J_{\mathrm{w}} / J_{\mathrm{s}}(\mathrm{CV}$, defined as the standard deviation divided by the arithmetic mean) for the analysis performed in Section 5.3. Only the results for characterizations carried out in four stages are presented.
As the $\mathrm{CV}$ of $J_{\mathrm{w}} / J_{\mathrm{s}}$ approaches zero, the normalized parameters converge towards unity (horizontal dashed line), signifying the membrane properties are more accurately predicted. This is due to the higher data quality expected at lower CVs. Fig. 6(B) shows the average normalized errors of the calculated water permeability, salt permeability, and structural parameter (blue, red, and green patterned bars, respectively) when the $\mathrm{CV}$ of the $J_{\mathrm{w}} / J_{\mathrm{s}}$ ratio within the four stages falls in the range indicated. The influence of the CV of $J_{\mathrm{w}} / J_{\mathrm{s}}$ on the eventual water and salt flux goodness of fit is also presented as $1-R^{2}$ in Fig. 6(B) (violet and orange patterned bars for $J_{\mathrm{w}}$ and $J_{\mathrm{s}}$ respectively). The percentage indicated above each group of columns represents the fraction of the entire sample that meets the CV criterion. Constancy of the reverse flux selectivity between the characterization stages gives not only better prediction of membrane parameters, but also enhances the fit of the calculated fluxes to the experimental readings. For example, when the coefficient of variation is less than $10 \%$, the calculated parameters deviate, on average, $10.8 \%$ from the true values and the coefficient of determination for both $J_{\mathrm{W}}$ and $J_{s}$ is greater than 0.975 . Similar trends were observed in the analysis of the two- and three-stage characterizations. Therefore, the $\mathrm{CV}$ of $J_{\mathrm{w}} / J_{\mathrm{s}}$ can be employed as a prerequisite for screening data reliability-conspicuous fluctuations would suggest large experimental errors beyond the tolerance of the algorithm.

The CVs of $J_{\mathrm{w}} / J_{\mathrm{s}}$ determined for the hand-cast TFC membranes and the commercial HTI-CTA, SW30 No PET, and Oasys TFC membranes range from 2.05 to $5.52 \%$ (Fig. 2 and Table 1). Based on the CV analysis, the deviation of the calculated parameters to their true values is approximately 7\% (Fig. 6(B)). The accuracy of the predictions, again, underscores the reliability of the proposed methodology to characterize forward osmosis membranes correctly. Additionally, the high $R_{\mathrm{w}}{ }^{2}$ and $R_{\mathrm{s}}{ }^{2}$ of the four membrane types (average of 0.991, Table 1) are in good agreement with the coefficients of determination of $\sim 0.992$ predicted by the analysis. This further emphasizes the validity of the utilizing the CVs of $J_{\mathrm{w}} / J_{\mathrm{s}}$ as a pre-condition for screening data integrity.

\subsection{Outlook and recommendations}

Analysis of the robustness and sensitivity of the proposed methodology suggests that a first requirement for its implementation is a consistent experimental value of $J_{\mathrm{w}} / J_{\mathrm{s}}$ between the various stages of each FO experiment. From our experience, careful experiments can ensure that the $\mathrm{CV}$ of the $J_{\mathrm{w}} / J_{\mathrm{s}}$ values lies below $3 \%$. We recommend a CV within $10 \%$ to confidently continue the
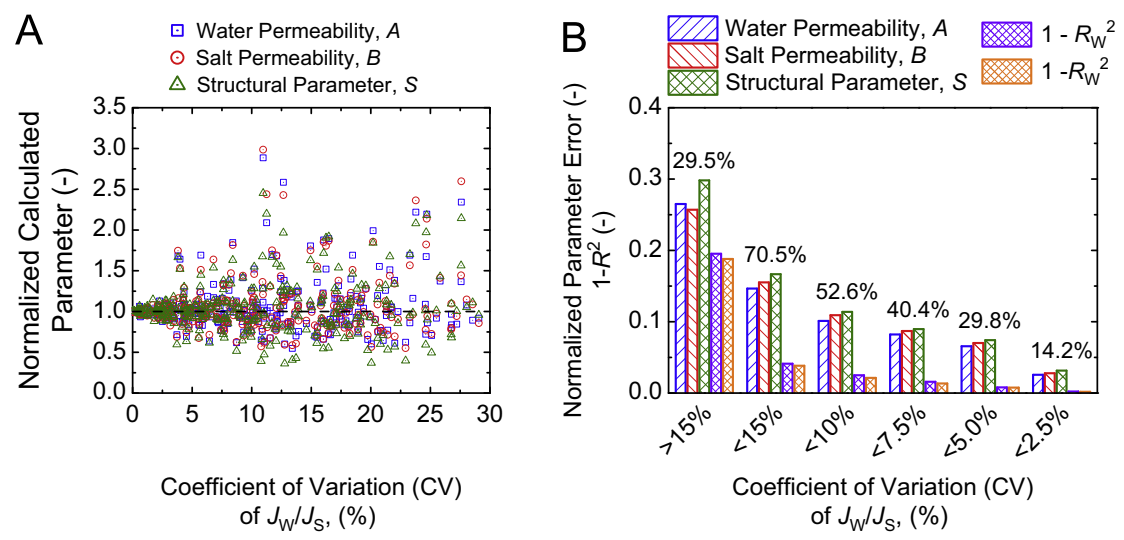

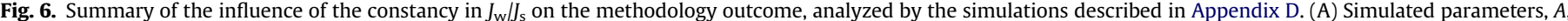

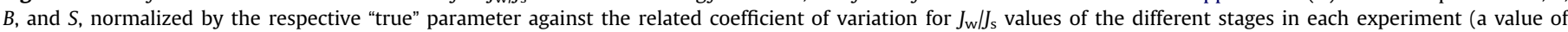

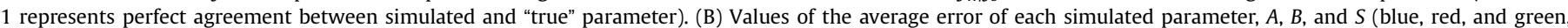

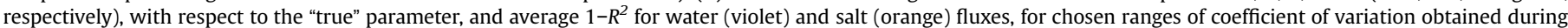

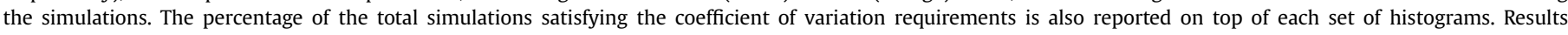

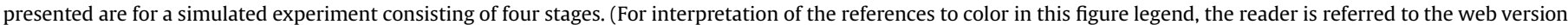
of this article.) 


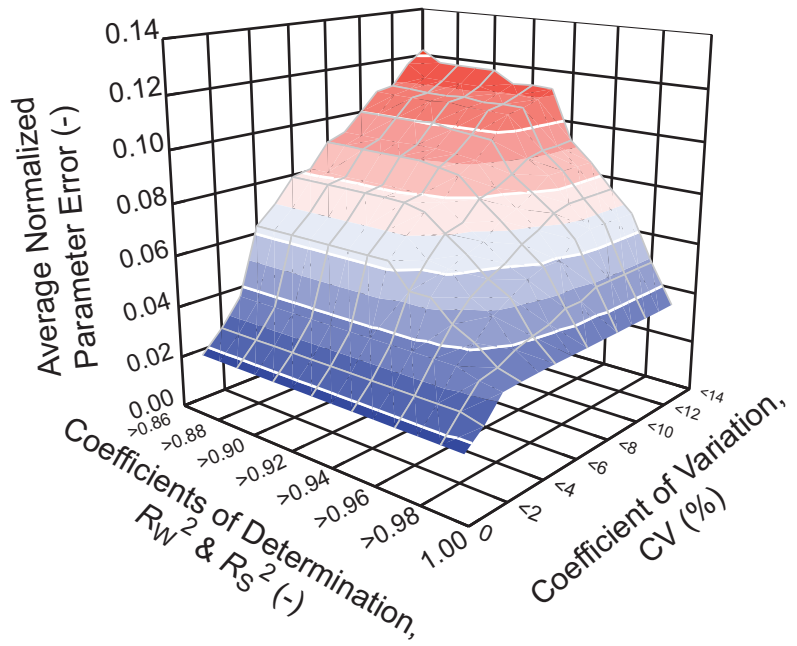

Fig. 7. 3-D surface plot of the normalized parameter error as a function of the coefficients of determination, $R_{\mathrm{w}}{ }^{2}$ and $R_{\mathrm{s}}{ }^{2}$, and of the coefficient of variation (CV) of $J_{\mathrm{w}} / J_{\mathrm{s}}$ for the four-stage characterization simulation described in Appendix D. The parameter error presented is the averaged normalized deviation of $A, B$, and $S$ from their "true" values for the samples that simultaneously meet the corresponding requirement for the coefficients of determination $R_{\mathrm{w}}{ }^{2}$ and $R_{\mathrm{s}}{ }^{2}$ (left horizontal axis) and for the coefficient of variation (right horizontal axis). Grid lines (gray) and contour lines (white) are included to guide the eyes.

analysis. When this number is higher than $15 \%$, the calculated membrane parameters may deviate significantly from their true values. A second control for the reliability of the results can be identified in the coefficients of determination for both water and salt flux. We recommend that both $R^{2}$ values be higher than 0.95 . When one or both fall below 0.9 , results should be taken cautiously.

Fig. 7 shows a surface plot of the average normalized parameter error as a function of the coefficients of determination (of water and salt fluxes) and the coefficient of variation (of $J_{\mathrm{w}} / J_{\mathrm{s}}$ values) for the four-stage characterization simulation discussed in Appendix D and Sections 5.3 and 5.4. An examination of the figure shows that attaining good $\mathrm{CV}$ and $R^{2}$ values concurrently is essential to minimize errors in the determined parameters. Meeting the dual conditions of CV of $J_{\mathrm{w}} / J_{\mathrm{s}}$ lesser than $10 \%$ and both $R_{\mathrm{w}}{ }^{2}$ and $R_{\mathrm{s}}{ }^{2}$ greater 0.95 will likely yield calculated parameters that are off by $10 \%$ or lower from their true values. Application of these two experimental and modeling checks is, therefore, necessary to achieve a reliable solution and to ensure high quality data. For this reason, results obtained by this method must always be accompanied by the reporting of all three values of: (i) the number of stages employed in the experiment, (ii) the observed $\mathrm{CV}$ between the $J_{w} / J_{s}$ ratios measured in the various stages, and (iii) the coefficients of determination calculated by the non-linear fitting procedure.

\section{Concluding remarks}

A simple protocol for characterization of the transport and structural parameters of FO membranes is presented, consisting of a single FO experiment divided into a number of stages, each utilizing a different draw solution concentration. The complete equations governing mass transport in FO are presented and used in combination with experimental measurements to determine the target parameters by an error minimization algorithm. The advantage of the proposed protocol, compared to the existing method comprising an RO experiment followed by FO, is demonstrated by the enhanced fitting of the predicted water and salt fluxes to the measured values for the four membrane types evaluated in this study. Thorough analysis indicates that the deviation of the calculated parameters from their true values is likely to be less than 3.5\%, further reinforcing the robustness of the protocol.

Notably, the values of membrane parameters obtained by the FO method were systematically different than those obtained by characterizing the same membranes using the existing approach. For all TFC membranes, the $A / B$ ratio measured by the FO methodology presented here was considerably lower (consistently between 30 and $50 \%$ ) than that determined by RO experiments. This observation casts doubt on the theoretical notion that membrane parameters are conserved in pressure- and osmotically-driven configurations. Regardless of the exact reasons for this disagreement, the difference in calculated membrane parameters and the greater prediction accuracy of the methodology presented here indicate that FO membranes should be characterized only by means of FO experiments. The development and the adoption of a standard and reliable characterization method for FO membranes are an important step to homogenize the results across different labs and to facilitate data exchange and interpretation.

\section{Acknowledgments}

We acknowledge the support received from the National Science Foundation under Award Number CBET 1232619. We also acknowledge the Graduate Fellowship (to N.Y.Y.) made by the Environment and Water Industrial Development Council of Singapore and the National Science Foundation Graduate Research Fellowship awarded to A.P.S.

\section{Appendix A. Derivation of the mass transport equations in FO}

A schematic of the salt concentration profile across a semipermeable membrane operating in FO mode is shown below. Due to the detrimental effects of internal concentration polarization (ICP) within the porous support, reverse salt permeation across the membrane, and external concentration polarization (ECP) in the feed solution, the osmotic driving force is lower than the osmotic pressure difference between the bulk draw and feed solutions.

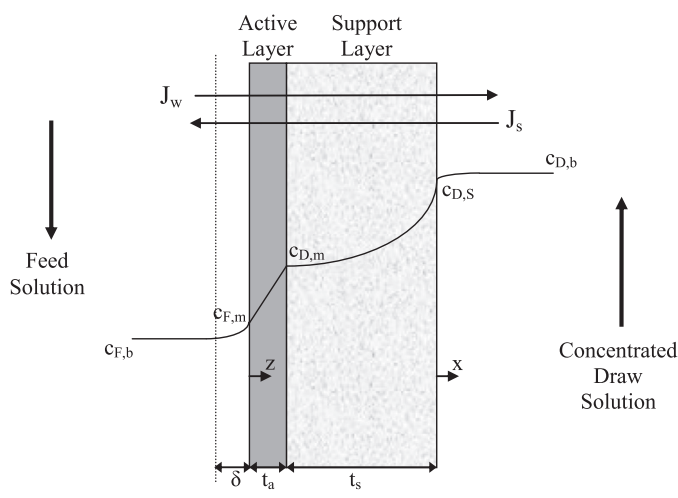

The water flux, $J_{\mathrm{w}}$, across the active layer is given by [42]

$J_{w}=A\left(\Delta \pi_{m}\right)$

where $\Delta \pi_{m}$ is the effective osmotic pressure difference across the active layer. This expression assumes that the reflection coefficient, $\sigma$, is equal to 1 and is therefore valid only for tight, saltrejecting membranes.

The reverse salt flux, $J_{s}$, across active layer is expressed by [40]

$-J_{S}=-B\left(c_{D, m}-c_{F, m}\right)$ 
Table B1

Complete set of FO experimental results.

\begin{tabular}{|c|c|c|c|c|c|c|c|c|}
\hline Membrane type & Membrane sample & Stage & $\boldsymbol{c}_{\mathbf{D}}(\mathrm{mM})$ & $\boldsymbol{c}_{\mathbf{F}}(\mathrm{mM})$ & $J_{\mathbf{w}}\left(\mathrm{L} \mathrm{m}^{-2} \mathrm{~h}^{-1}\right)$ & $J_{\mathbf{s}}\left(\mathrm{mmol} \mathrm{m}{ }^{-2} \mathrm{~h}^{-1}\right)$ & $\boldsymbol{J}_{\mathrm{w}} / \boldsymbol{J}_{\mathbf{s}}\left(\mathrm{L} \mathrm{mol}^{-1}\right)$ & CV $(\%)$ \\
\hline \multirow[t]{12}{*}{ Hand-Cast TFC } & \#A1 & 1 & 255 & 0.14 & 9.6 & 28.7 & 334 & 2.05 \\
\hline & & 2 & 341 & 0.19 & 11.5 & 33.6 & 341 & \\
\hline & & 3 & 465 & 0.28 & 13.5 & 38.8 & 348 & \\
\hline & & 4 & 642 & 0.34 & 15.5 & 46.5 & 333 & \\
\hline & \#A2 & 1 & 258 & 0.07 & 9.4 & 28.9 & 326 & 3.57 \\
\hline & & 2 & 420 & 0.13 & 12.1 & 37.0 & 326 & \\
\hline & & 3 & 623 & 0.19 & 14.9 & 47.8 & 312 & \\
\hline & & 4 & 844 & 0.27 & 17.3 & 57.2 & 303 & \\
\hline & \#A3 & 1 & 329 & 0.10 & 9.3 & 40.9 & 228 & 3.84 \\
\hline & & 2 & 489 & 0.18 & 12.3 & 49.3 & 249 & \\
\hline & & 3 & 689 & 0.26 & 14.0 & 60.2 & 233 & \\
\hline & & 4 & 911 & 0.35 & 16.3 & 68.2 & 238 & \\
\hline \multirow[t]{12}{*}{ HTI-CTA } & \#B1 & 1 & 304 & 0.10 & 8.0 & 195.3 & 41 & 5.52 \\
\hline & & 2 & 577 & 0.34 & 11.3 & 259.5 & 44 & \\
\hline & & 3 & 916 & 0.70 & 14.1 & 302.0 & 47 & \\
\hline & & 4 & 1517 & 1.21 & 17.9 & 399.8 & 45 & \\
\hline & \#B2 & 1 & 302 & 0.11 & 7.7 & 145.8 & 53 & 5.44 \\
\hline & & 2 & 502 & 0.30 & 10.4 & 188.7 & 55 & \\
\hline & & 3 & 852 & 0.54 & 13.6 & 241.7 & 56 & \\
\hline & & 4 & 1461 & 0.95 & 17.7 & 294.1 & 60 & \\
\hline & \#B3 & 1 & 325 & 0.19 & 9.2 & 205.0 & 45 & 4.10 \\
\hline & & 2 & 565 & 0.44 & 11.6 & 260.0 & 45 & \\
\hline & & 3 & 891 & 0.72 & 14.6 & 315.4 & 46 & \\
\hline & & 4 & 1454 & 1.09 & 18.3 & 373.8 & 49 & \\
\hline \multirow[t]{12}{*}{ SW30 No PET } & \#C1 & 1 & 404 & 0.07 & 5.4 & 27.8 & 193 & 5.43 \\
\hline & & 2 & 682 & 0.14 & 6.3 & 33.9 & 185 & \\
\hline & & 3 & 1002 & 0.19 & 7.3 & 39.0 & 187 & \\
\hline & & 4 & 1581 & 0.29 & 7.9 & 46.7 & 170 & \\
\hline & $\# C 2$ & 1 & 404 & 0.22 & 5.1 & 18.8 & 269 & 2.23 \\
\hline & & 2 & 688 & 0.28 & 6.0 & 22.5 & 268 & \\
\hline & & 3 & 1013 & 0.33 & 6.9 & 25.6 & 268 & \\
\hline & & 4 & 1582 & 0.39 & 8.0 & 31.1 & 257 & \\
\hline & \#C3 & 1 & 405 & 0.18 & 5.3 & 20.6 & 259 & 2.54 \\
\hline & & 2 & 691 & 0.25 & 6.7 & 24.7 & 271 & \\
\hline & & 3 & 1029 & 0.32 & 7.6 & 28.0 & 270 & \\
\hline & & 4 & 1582 & 0.50 & 8.5 & 32.9 & 259 & \\
\hline \multirow[t]{12}{*}{ Oasys TFC } & \#D1 & 1 & 137 & 0.09 & 11.2 & 66.8 & 167 & 5.28 \\
\hline & & 2 & 272 & 0.20 & 14.5 & 92.9 & 156 & \\
\hline & & 3 & 576 & 0.36 & 20.0 & 125.8 & 159 & \\
\hline & & 4 & 1060 & 0.62 & 24.9 & 169.0 & 147 & \\
\hline & \#D2 & 1 & 174 & 0.10 & 9.2 & 67.4 & 137 & 3.48 \\
\hline & & 2 & 324 & 0.24 & 12.4 & 85.8 & 145 & \\
\hline & & 3 & 644 & 0.38 & 16.5 & 112.3 & 147 & \\
\hline & & 4 & 1191 & 0.58 & 20.4 & 137.4 & 148 & \\
\hline & \#D3 & 1 & 154 & 0.05 & 10.2 & 65.7 & 155 & 4.84 \\
\hline & & 2 & 298 & 0.15 & 14.0 & 86.5 & 162 & \\
\hline & & 3 & 607 & 0.30 & 18.8 & 113.6 & 166 & \\
\hline & & 4 & 1084 & 0.50 & 23.2 & 133.2 & 174 & \\
\hline
\end{tabular}

As water permeates across the membrane, it dilutes the draw solution in the support layer, resulting in dilutive internal concentration polarization, ICP [32]. Diffusion works to restore this local concentration to the bulk draw solution concentration, but it is hindered by the porous support, which acts as an unstirred boundary layer. The salt flux across the porous support is the sum of the diffusive component, driven by the salt concentration gradient, and the convective component, arising from the permeation of water through the membrane

$-J_{s}=-D^{S} \frac{d c(x)}{d x}+J_{w} c(x)$

where $D^{s}$ is the effective diffusion coefficient of the draw solute in the porous support. The latter can be related to the bulk diffusion coefficient, $D$, by accounting for the porosity, $\varepsilon$, and tortuosity, $\tau$, of the support layer, i.e., $D^{s}=D \varepsilon / \tau[40]$.
At steady-state, the salt fluxes across the active (Eq. (A2)) and support (Eq. (A3)) layers are equal:

$\frac{d c(x)}{d x}-\frac{J_{w}}{D^{S}} c(x)=\frac{B}{D^{S}}\left(c_{D, m}-c_{F, m}\right)$

Integrating Eq. (A4) across the support layer thickness, from the porous layer draw solution interface, $x=0$, where the salt concentration is $c_{D, s}$, to the porous layer-active layer interface, $x=-t_{s}$, where the salt concentration is $c_{D, m}$ (Fig. 1), yields

$c_{D, m}=c_{D, S} \exp \left(-\frac{J_{w} S}{D}\right)+\frac{B}{J_{w}}\left(c_{D, m}-c_{F, m}\right)\left[\exp \left(-\frac{J_{w} S}{D}\right)-1\right]$

where $S=t_{S} \tau / \varepsilon$ is the support layer structural parameter [32]. Eq. (A5) indicates that the salt concentration at the active-support interface, $c_{D, m}$, is the sum of two terms. The effect of dilutive ICP is described by the first term on the right-hand side. The second term accounts for the decrease in salt concentration at the 
Table C1

Complete set of calculated parameters using both algorithms (Excel and Matlab).

\begin{tabular}{|c|c|c|c|c|c|c|c|c|c|c|c|c|c|c|}
\hline \multirow[t]{2}{*}{ Membrane type } & \multirow[t]{2}{*}{ Sample } & \multicolumn{5}{|c|}{ Microsoft Excel (Visual Basic) } & \multicolumn{5}{|l|}{ Mathworks Matlab } & \multicolumn{3}{|c|}{ Deviation } \\
\hline & & $\boldsymbol{A}\left(\mathrm{L} \mathrm{m}^{-2} \mathrm{~h}^{-1} \mathrm{bar}^{-1}\right)$ & $\boldsymbol{B}\left(\mathrm{L} \mathrm{m}^{-2} \mathrm{~h}^{-1}\right)$ & $\boldsymbol{S}(\mu \mathrm{m})$ & $R^{2}\left[J_{w}\right]$ & $R^{2}\left[J_{s}\right]$ & $\boldsymbol{A}\left(\mathrm{L} \mathrm{m}^{-2} \mathrm{~h}^{-1} \mathrm{bar}^{-1}\right)$ & $\boldsymbol{B}\left(\mathrm{L} \mathrm{m}^{-2} \mathrm{~h}^{-1}\right)$ & $\boldsymbol{S}(\mu \mathrm{m})$ & $R^{2}\left[J_{w}\right]$ & $R^{2}\left[J_{s}\right]$ & $\boldsymbol{A}(\%)$ & $\boldsymbol{B}(\%)$ & $\boldsymbol{S}(\%)$ \\
\hline \multirow[t]{3}{*}{ Hand-cast TFC } & \#A1 & 1.61 & 0.235 & 400 & 0.998 & 0.996 & 1.60 & 0.234 & 398 & 0.998 & 0.996 & 0.52 & 0.43 & 0.38 \\
\hline & \#A2 & 1.23 & 0.196 & 328 & 0.994 & 0.990 & 1.23 & 0.195 & 327 & 0.994 & 0.990 & 0.18 & 0.34 & 0.32 \\
\hline & \#A3 & 1.21 & 0.252 & 395 & 0.992 & 0.997 & 1.21 & 0.252 & 394 & 0.992 & 0.997 & 0.24 & 0.12 & 0.21 \\
\hline \multirow[t]{3}{*}{ HTI-CTA } & \#B1 & 1.22 & 1.35 & 481 & 0.997 & 0.976 & 1.21 & 1.35 & 480 & 0.997 & 0.976 & 0.66 & 0.27 & 0.21 \\
\hline & \#B2 & 1.17 & 1.00 & 473 & 0.994 & 0.992 & 1.16 & 1.00 & 472 & 0.994 & 0.991 & 0.56 & 0.22 & 0.22 \\
\hline & \#B3 & 1.63 & 1.73 & 541 & 0.989 & 0.993 & 1.63 & 1.72 & 540 & 0.989 & 0.993 & 0.20 & 0.53 & 0.17 \\
\hline \multirow[t]{3}{*}{ SW30 No PET } & $\# C 1$ & 1.32 & 0.361 & 1620 & 0.963 & 0.982 & 1.31 & 0.359 & 1619 & 0.962 & 0.983 & 0.62 & 0.43 & 0.03 \\
\hline & \#C2 & 1.05 & 0.197 & 1540 & 0.994 & 0.983 & 1.04 & 0.196 & 1533 & 0.994 & 0.984 & 0.66 & 0.51 & 0.43 \\
\hline & \#C3 & 1.27 & 0.239 & 1510 & 0.996 & 0.994 & 1.27 & 0.238 & 1503 & 0.996 & 0.994 & 0.02 & 0.41 & 0.48 \\
\hline \multirow[t]{3}{*}{ Oasys TFC } & \#D1 & 3.69 & 1.19 & 424 & 0.992 & 0.989 & 3.68 & 1.19 & 423 & 0.992 & 0.989 & 0.39 & 0.44 & 0.16 \\
\hline & \#D2 & 3.37 & 1.14 & 589 & 0.998 & 0.997 & 3.37 & 1.14 & 589 & 0.999 & 0.997 & 0.11 & 0.16 & 0.06 \\
\hline & \#D3 & 4.26 & 1.26 & 526 & 0.996 & 0.993 & 4.24 & 1.25 & 524 & 0.996 & 0.993 & 0.43 & 0.57 & 0.31 \\
\hline
\end{tabular}

membrane interface due to the reverse permeation of draw solution salt into the active layer.

As water permeates across the membrane, the feed solutes are selectively retained by the semi-permeable active layer and build up within the boundary layer at the active side, resulting in concentrative ECP. Similar to ICP, the salt flux within this ECP boundary layer comprises diffusive and convective components

$-J_{s}=-D \frac{d c(z)}{d z}+J_{w} c(z)$

At steady-state, the salt flux within the ECP boundary layer (Eq. (A6)) is equal to the salt flux across the active layer (Eq. (A2)). Integrating the resulting equation across the ECP boundary layer from the active layer, $z=0$, where the salt concentration is $c_{F, m}$, to the bulk draw solution, $z=-\delta$, where the salt concentration is $c_{F, b}$, yields

$c_{F, m}=c_{F, b} \exp \left(\frac{J_{w}}{k}\right)-\frac{B}{J_{w}}\left(c_{D, m}-c_{F, m}\right)\left[1-\exp \left(\frac{J_{W}}{k}\right)\right]$

where $k=D / \delta$ is the boundary layer mass transfer coefficient. Inspecting Eq. (A7) reveals that $c_{F, m}$ is dependent on two terms. The first term describes the bulk feed concentration, $c_{F, b}$, corrected for concentrative ECP by the factor $\exp \left(J_{\mathrm{w}} / k\right)$, while the second term represents the increase in salt concentration due to draw solute leakage across the active layer.

Both $c_{D, m}$ and $c_{F, m}$ are local interfacial concentrations on either side of the active layer interface and therefore are not experimentally accessible. To circumvent this, we subtract Eq. (A5) from Eq. (A7) and rearrange to obtain

$c_{D, m}-c_{F, m}=\frac{c_{D, S} \exp \left(-\frac{J_{W} S}{D}\right)-c_{F, b} \exp \left(\frac{J_{W}}{k}\right)}{1+\left(B / J_{W}\right)\left[\exp \left(\frac{J_{W}}{k}\right)-\exp \left(-\frac{J_{W} S}{D}\right)\right]}$

Next, we assume that the osmotic pressure is linearly proportional to the salt concentration; i.e., the van't Hoff equation is applicable. Hence, the effective osmotic driving force, $\Delta \pi_{m}$, is proportional to $\Delta c_{m}=c_{D, m}-c_{F, m}$ (Eq. (A8)). In the analysis, we also assume that ECP in the draw solution is negligible because the support layer thickness is relatively large, thereby dominating concentration polarization, i.e., $\pi_{D, S} \cong \pi_{D, b}$. Substituting $\Delta \pi_{m}$ into Eq. (A1) yields an expression for the water flux in $\mathrm{FO}$

$J_{w}=A\left\{\frac{\pi_{D, b} \exp \left(-\frac{J_{W} S}{D}\right)-\pi_{F, b} \exp \left(\frac{J_{W}}{k}\right)}{1+\frac{B}{J_{w}}\left[\exp \left(\frac{J_{w}}{k}\right)-\exp \left(-\frac{J_{W} S}{D}\right)\right]}\right\}$
Table D1

Water permeability, $A$, salt permeability, $B$, and structural parameter, $S$, assigned to the model membrane employed in this analysis.

\begin{tabular}{lll}
\hline $\begin{array}{l}\text { Water permeability, } \boldsymbol{A} \\
\left(\mathrm{L} \mathrm{m}^{-2} \mathrm{~h}^{-1} \mathrm{bar}^{-1}\right)\end{array}$ & $\begin{array}{l}\text { Salt permeability, } \boldsymbol{B} \\
\left(\mathrm{L} \mathrm{m}^{-2} \mathrm{~h}^{-1}\right)\end{array}$ & $\begin{array}{l}\text { Structural } \\
\text { parameter, } \boldsymbol{S}(\mu \mathrm{m})\end{array}$ \\
\hline 1.23 & 0.196 & 328
\end{tabular}

Table D2

Salt concentrations, $c$, and osmotic pressures, $\pi$, of the draw and feed solutions at the three stages of the characterization experiment, and the corresponding "true" water flux, $J_{\mathrm{w}}$, and reverse salt flux, $J_{\mathrm{s}}$.

\begin{tabular}{lllllll}
\hline Stage & $\boldsymbol{c}_{\mathbf{D}}(\mathrm{mM})$ & $\boldsymbol{\pi}_{\mathbf{D}}($ bar $)$ & $\boldsymbol{c}_{\mathbf{F}}(\mathrm{mM})$ & $\boldsymbol{\pi}_{\mathbf{F}}($ bar $)$ & $\boldsymbol{J}_{\mathbf{w}}\left(\mathrm{L} \mathrm{m}^{-2} \mathrm{~h}^{-1}\right)$ & $\boldsymbol{J}_{\mathbf{s}}\left(\mathrm{mmol} \mathrm{m}^{-2} \mathrm{~h}^{-1}\right)$ \\
\hline 1 & 258 & 12.78 & 0.07 & 0.00 & 8.92 & 28.7 \\
2 & 420 & 20.81 & 0.13 & 0.01 & 12.01 & 38.6 \\
3 & 623 & 30.87 & 0.19 & 0.01 & 14.90 & 47.9 \\
4 & 844 & 41.82 & 0.27 & 0.01 & 17.35 & 55.8 \\
\hline
\end{tabular}

Substituting $\Delta c_{m}$ into Eq. (A2) yields an expression for the reverse salt flux in FO

$J_{S}=B\left\{\frac{c_{D, b} \exp \left(-\frac{J_{W} S}{D}\right)-c_{F, b} \exp \left(\frac{J_{W}}{k}\right)}{1+\frac{B}{J_{w}}\left[\exp \left(\frac{J_{W}}{k}\right)-\exp \left(-\frac{J_{W} S}{D}\right)\right]}\right\}$

These equations utilize experimentally accessible parameters and incorporate the performance-limiting phenomena of ICP and ECP as well as salt leakage across the membrane.

\section{Appendix B}

See Table B1 for complete set of FO experimental results.

\section{Appendix C}

See Table C1 for complete set of calculated parameters using both algorithms (Excel and Matlab).

\section{Appendix D. Protocol to evaluate the robustness of the proposed methodology}

The membrane characterization experiments entail measurement of the water and salt fluxes, and the draw and feed solution 
Table D3

An example of the uncertainty deliberately introduced in the flux measurements. Here, the root-mean-square of the error introduced to the water fluxes, $J_{\mathrm{w}}$, and salt fluxes, $J_{s}$, works out to be $2.5 \%$.

\begin{tabular}{|c|c|c|c|c|c|}
\hline \multicolumn{2}{|l|}{ True } & \multicolumn{2}{|l|}{ Offset } & \multicolumn{2}{|c|}{$\begin{array}{l}\text { Relative } \\
\text { difference }\end{array}$} \\
\hline $\begin{array}{l}\boldsymbol{J}_{\mathbf{w}} \\
\left(\mathrm{L} \mathrm{m}^{-2} \mathrm{~h}^{-1}\right)\end{array}$ & $\begin{array}{l}\boldsymbol{J}_{\mathbf{s}} \\
\left(\mathrm{mmol} \mathrm{m}{ }^{-2} \mathrm{~h}^{-1}\right)\end{array}$ & $\begin{array}{l}\boldsymbol{J}_{\mathbf{w}} \\
\left(\mathrm{L} \mathrm{m}^{-2} \mathrm{~h}^{-1}\right)\end{array}$ & $\begin{array}{l}\boldsymbol{J}_{\mathbf{s}} \\
\left(\mathrm{mmol} \mathrm{m}^{-2} \mathrm{~h}^{-1}\right)\end{array}$ & $J_{\mathbf{w}}(\%)$ & $J_{\mathbf{s}}(\%)$ \\
\hline 8.92 & 28.7 & 9.04 & 28.1 & 1.37 & -2.17 \\
\hline 12.01 & 38.6 & 11.64 & 37.9 & -3.06 & -1.77 \\
\hline 14.90 & 47.9 & 15.03 & 46.3 & 0.87 & -3.43 \\
\hline 17.35 & 55.8 & 16.81 & 57.4 & -3.14 & 2.92 \\
\hline
\end{tabular}

concentrations. To probe the robustness of the methodology to inevitable measurement uncertainties, errors in water and salt flux measurements were deliberately introduced to the characterization of a model membrane. The calculated $A, B$, and $S$ values, using the simulated flux readings (i.e., those in which random errors were deliberately introduced), were compared to the actual membrane parameters to examine the effect of random measurement errors.

Typical water permeability, salt permeability, and structural parameter values of a high performance polyamide TFC membrane (indicated in Table D1) were considered as the "true" values during the assessment of the effect of random errors in the flux measurements. Salt concentrations representative of a typical characterization experiment were chosen for the draw and feed solution at each stage. The water and salt fluxes at each stage were determined using the "true" membrane characteristic parameters and the governing equations (Eqs. (1) and (2)) for $J_{\mathrm{w}}$ and $J_{\mathrm{s}}$. These "true" water and salt fluxes are shown in Table D2 for a four-stage characterization experiment, together with the draw and feed solution concentrations and osmotic pressures.

To simulate experimental uncertainty, measurement errors were systematically introduced to the water and salt flux values. Deviations of the eight $J_{\mathrm{w}}$ and $J_{\mathrm{s}}$ readings from the true values of the four stage experiment were randomly generated using a computer algorithm. The overall error introduced to the experiment was calculated by taking the root-mean-square of the relative differences (defined as the difference between the erroneous flux and the true flux, divided by the true value). An example of the offset fluxes is presented in Table D3 using the true water and salt fluxes determined above. In this illustration, the measurement error is $2.5 \%$, calculated from the root-meansquare of the eight $J_{\mathrm{w}}$ and $J_{\mathrm{s}}$ relative differences.

The offset "experimental" values of water and salt fluxes, together with the concentrations and osmotic pressures in Table $\mathrm{D} 2$, were then input into the Excel code described in Section 3 to determine the combination of membrane parameters $A, B$, and $S$ which best fits the data. The coefficients of determination, $R^{2}$, for both water and salt fluxes, and the coefficient of variation of $J_{\mathrm{w}} / J_{\mathrm{s}}$ over the four stages were also calculated. For example, the above offset flux values would yield $A=1.16 \mathrm{~L} \mathrm{~m}^{-2} \mathrm{~h}^{-1} \mathrm{bar}^{-1}$, $B=0.187 \mathrm{~L} \mathrm{~m}^{-2} \mathrm{~h}^{-1}$, and $S=321 \mu \mathrm{m}$.

To examine the effect of the magnitude of measurement uncertainty on the calculated membrane parameters, root-mean-square errors ranging from 0 to $15 \%$, in $0.1 \%$ intervals, were introduced to the $J_{\mathrm{w}}$ and $J_{\mathrm{s}}$ values to yield 151 hypothetical experimental runs. When creating the "experimental" fluxes, the randomly generated errors can potentially skew the average towards higher or lower fluxes. To eliminate this potential bias, a mirror-image of the dataset was added. That is, another 151 hypothetical experimental runs were added to the population, with same magnitude of error as in the initial 151 samples, but with opposite sign. This ensures that the arithmetic means of the water and salt fluxes of the total 302 samples were exactly equal to the true $J_{\mathrm{w}}$ and $J_{\mathrm{s}}$ values and any positive or negative bias of the relative difference was eliminated.

The analytical exercise described here was repeated for hypothetical characterization experiments consisting of two and three stages, using the same model membrane parameters. The results are presented in Section 5.3, and the influence of the number of stages on the accuracy of the proposed methodology is discussed. Section 5.4 looks at how fluctuations in the water flux to reverse salt flux ratio between stages of the characterization protocol influence the accuracy of the membrane properties predictions. In Section 5.5, we draw on these findings to recommend the range of experimental uncertainties the proposed methodology can tolerate while still yielding reasonably accurate membrane parameter calculations.

\section{Appendix E. Supporting information}

The Excel spreadsheet and the Matlab file described in Section 3.2 to calculate the membrane properties are available for download, gratis for non-commercial use, from the Supplementary materials of the online version at http://dx.doi.org/10. 1016/j.memsci.2013.05.023. Instruction on using the files can be found online at http://www.yale.edu/env/elimelech/Research_Page/FO_characterization.html.

\section{Nomenclature}

A water permeability coefficient of the membrane active layer membrane area

$A_{R O} \quad$ water permeability coefficient measured in RO experiments

$B \quad$ solute permeability coefficient of the membrane active layer

$B_{R O} \quad$ solute permeability coefficient measured in RO experiments

solute concentration

$\Delta c \quad$ solute concentration difference

CV coefficient of variation

$D \quad$ bulk solute diffusion coefficient

$D^{S} \quad$ effective solute diffusion coefficient in the membrane support layer $=D \varepsilon / \tau$

E global error from the least-squared minimization procedure

stage number: $i=1,2,3,4$

flux

average flux

mass transfer coefficient

resistance to solute diffusion in the membrane support layer

coefficient of determination

gas constant

structural parameter of the membrane support layer

$S_{R O+F O} \quad$ structural parameter obtained by current approach comprising an RO experiment followed by an FO experiment

$S S_{\text {err }} \quad$ residual sum of squares

$S S_{\text {TOT }}$ total sum of squares

$T$

time

absolute temperature

thickness of the membrane active layer

thickness of the membrane support layer

volume 
$x \quad$ coordinate within the height of the draw solution channel

Z coordinate within the membrane thickness

\section{Greek symbols}

$\delta \quad$ thickness of the feed solution boundary layer

$\varepsilon \quad$ porosity of the membrane support layer

$\pi \quad$ osmotic pressure

$\Delta \pi \quad$ osmotic pressure difference

$\sigma \quad$ reflection coefficient

$\tau \quad$ tortuosity of the membrane support layer

$v \quad$ total number of species

\section{Superscripts}

$\begin{array}{ll}\text { CALC } & \begin{array}{l}\text { calculated by least-squared minimization } \\ \text { procedure }\end{array} \\ \text { EXP } & \text { measured experimentally } \\ n & \text { total number of stages }\end{array}$

\section{Subscripts}

$\begin{array}{ll}0 & \text { initial condition } \\ b & \text { solution bulk } \\ D & \text { draw solution } \\ F & \text { feed solution } \\ i & \text { stage number } \\ m & \text { membrane active layer interface } \\ S & \text { solute species } \\ S & \text { membrane support layer interface } \\ w & \text { water species }\end{array}$

\section{References}

[1] T.Y. Cath, A.E. Childress, M. Elimelech, Forward osmosis: principles, applications, and recent developments, J. Membrane Sci. 281 (2006) 70-87.

[2] R.W. Holloway, A.E. Childress, K.E. Dennett, T.Y. Cath, Forward osmosis for concentration of anaerobic digester centrate, Water Res. 41 (2007) 4005-4014.

[3] W. Tang, H.Y. Ng, Concentration of brine by forward osmosis: performance and influence of membrane structure, Desalination 224 (2008) 143-153.

[4] A. Achilli, T.Y. Cath, E.A. Marchand, A.E. Childress, The forward osmosis membrane bioreactor: a low fouling alternative to MBR processes, Desalination 239 (2009) 10-21.

[5] E.M. Garcia-Castello, J.R. McCutcheon, M. Elimelech, Performance evaluation of sucrose concentration using forward osmosis, J. Membrane Sci. 338 (2009) 61-66.

[6] B. Jiao, A. Cassano, E. Drioli, Recent advances on membrane processes for the concentration of fruit juices: a review, J. Food Eng. 63 (2004) 303-324.

[7] Q. Yang, K.Y. Wang, T.S. Chung, A novel dual-layer forward osmosis membrane for protein enrichment and concentration, Separ. Purif. Technol. 69 (2009) 269-274.

[8] R.E. Kravath, J.A. Davis, Desalination of sea-water by direct osmosis, Desalination 16 (1975) 151-155.

[9] M. Elimelech, W.A. Phillip, The future of seawater desalination: energy, technology, and the environment, Science 333 (2011) 712-717.

[10] J.L. Cartinella, T.Y. Cath, M.T. Flynn, G.C. Miller, K.W. Hunter, A.E. Childress, Removal of natural steroid hormones from wastewater using membrane contactor processes, Environ. Sci. Technol. 40 (2006) 7381-7386.

[11] T.Y. Cath, D. Adams, A.E. Childress, Membrane contactor processes for wastewater reclamation in space II. Combined direct osmosis, osmotic distillation, and membrane distillation for treatment of metabolic wastewater, J. Membrane Sci. 257 (2005) 111-119.

[12] T.Y. Cath, S. Gormly, E.G. Beaudry, M.T. Flynn, V.D. Adams, A.E. Childress, Membrane contactor processes for wastewater reclamation in space. Part Direct osmotic concentration as pretreatment for reverse osmosis, J. Membrane Sci. 257 (2005) 85-98.

[13] L.A. Hoover, W.A. Phillip, A. Tiraferri, N.Y. Yip, M. Elimelech, Forward with osmosis: emerging applications for greater sustainability, Environ. Sci. Technol. 45 (2011) 9824-9830.
[14] N.N. Bui, M.L. Lind, E.M.V. Hoek, J.R. McCutcheon, Electrospun nanofiber supported thin film composite membranes for engineered osmosis, J. Membrane Sci. 385 (2011) 10-19.

[15] B.D. Coday, D.M. Heil, P. Xu, T. Cath, Effects of transmembrane hydraulic pressure on performance of forward osmosis membranes, Environ. Sci. Technol. 47 (2013) 2386-2393.

[16] C. Qiu, L. Setiawan, R. Wang, C.Y.Y. Tang, A.G. Fane, High performance flat sheet forward osmosis membrane with an NF-like selective layer on a woven fabric embedded substrate, Desalination 287 (2012) 266-270.

[17] C.Q. Qiu, S.R. Qi, C.Y.Y. Tang, Synthesis of high flux forward osmosis membranes by chemically crosslinked layer-by-layer polyelectrolytes, J. Membrane Sci. 381 (2011) 74-80.

[18] Q. Saren, C.Q. Qiu, C.Y.Y. Tang, Synthesis and characterization of novel forward osmosis membranes based on layer-by-layer assembly, Environ. Sci. Technol. 45 (2011) 5201-5208.

[19] L. Setiawan, R. Wang, K. Li, A.G. Fane, Fabrication of novel poly(amide-imide) forward osmosis hollow fiber membranes with a positively charged nanofiltration-like selective layer, J. Membrane Sci. 369 (2011) 196-205.

[20] J.C. Su, T.S. Chung, B.J. Helmer, J.S. de Wit, Enhanced double-skinned FO membranes with inner dense layer for wastewater treatment and macromolecule recycle using sucrose as draw solute, J. Membrane Sci. 396 (2012) 92-100.

[21] J.C. Su, Q. Yang, J.F. Teo, T.S. Chung, Cellulose acetate nanofiltration hollow fiber membranes for forward osmosis processes, J. Membrane Sci. 355 (2010) 36-44.

[22] A. Tiraferri, N.Y. Yip, W.A. Phillip, J.D. Schiffman, M. Elimelech, Relating performance of thin-film composite forward osmosis membranes to support layer formation and structure, J. Membrane Sci. 367 (2011) 340-352.

[23] K.Y. Wang, R.C. Ong, T.S. Chung, Double-skinned forward osmosis membranes for reducing internal concentration polarization within the porous sublayer, Ind. Eng. Chem. Res. 49 (2010) 4824-4831.

[24] R. Wang, L. Shi, C.Y.Y. Tang, S.R. Chou, C. Qiu, A.G. Fane, Characterization of novel forward osmosis hollow fiber membranes, J. Membrane Sci. 355 (2010) 158-167.

[25] J. Wei, C.Q. Qiu, C.Y.Y. Tang, R. Wang, A.G. Fane, Synthesis and characterization of flat-sheet thin film composite forward osmosis membranes, J. Membrane Sci. 372 (2011) 292-302.

[26] N. Widjojo, T.S. Chung, M. Weber, C. Maletzko, V. Warzelhan, The role of sulphonated polymer and macrovoid-free structure in the support layer for thin-film composite (TFC) forward osmosis (FO) membranes, J. Membrane Sci. 383 (2011) 214-223.

[27] Q. Yang, K.Y. Wang, T.S. Chung, Dual-layer hollow fibers with enhanced flux as novel forward osmosis membranes for water production, Environ. Sci. Technol. 43 (2009) 2800-2805.

[28] S. Zhang, K.Y. Wang, T.S. Chung, H.M. Chen, Y.C. Jean, G. Amy, Well-constructed cellulose acetate membranes for forward osmosis: minimized internal concentration polarization with an ultra-thin selective layer, J. Membrane Sci. 360 (2010) 522-535.

[29] N.Y. Yip, A. Tiraferri, W.A. Phillip, J.D. Schiffman, M. Elimelech, High performance thin-film composite forward osmosis membrane, Environ. Sci. Technol. 44 (2010) 3812-3818.

[30] A. Tiraferri, Y. Kang, E.P. Giannelis, M. Elimelech, Highly hydrophilic thin-film composite forward osmosis membranes functionalized with surface-tailored nanoparticles, ACS Appl. Mater. Interfaces 4 (2012) 5044-5053.

[31] A. Tiraferri, Y. Kang, E.P. Giannelis, M. Elimelech, Superhydrophilic thin-film composite forward osmosis membranes for organic fouling control: fouling behavior and antifouling mechanisms, Environ. Sci. Technol. 46 (2012) 11135-11144.

[32] J.R. McCutcheon, M. Elimelech, Modeling water flux in forward osmosis: Implications for improved membrane design, Aiche J. 53 (2007) 1736-1744.

[33] T.Y. Cath, M. Elimelech, J.R. McCutcheon, R.L. McGinnis, A. Achilli, D. Anastasio, A.R. Brady, A.E. Childress, I.V. Farr, N.T. Hancock, J. Lampi, L.D. Nghiem, M. Xie, N.Y. Yip, Standard methodology for evaluating membrane performance in osmotically driven membrane processes, Desalination 312 (2013) 31-38.

[34] B.D. Coday, D.M. Heil, P. Xu, T. Cath, Effects of transmembrane hydraulic pressure on performance of forward osmosis membranes, Environ. Sci. Technol. 47 (2013) 2386-2393.

[35] N.Y. Yip, A. Tiraferri, W.A. Phillip, J.D. Schiffman, L.A. Hoover, Y.C. Kim, M. Elimelech, Thin-film composite pressure retarded osmosis membranes for sustainable power generation from salinity gradients, Environ. Sci. Technol. 45 (2011) 4360-4369.

[36] V. Vitagliano, P.A. Lyons, Diffusion coefficients for aqueous solutions of sodium chloride and barium chloride, J. Am. Chem. Soc. 78 (1956) 1549-1552.

[37] D. Fylstra, L. Lasdon, J. Watson, A. Waren, Design and use of the Microsoft Excel Solver, Interfaces 28 (1998) 29-55.

[38] E. Isaacson, H.B. Keller, Analysis of Numerical Methods, Dover Publications, 1994

[39] O. Kedem, A. Katchalsky, Physical interpretation of phenomenological coefficients of membrane permeability, J. Gen. Physiol. 45 (1961) 143 -\&.

[40] W.A. Phillip, J.S. Yong, M. Elimelech, Reverse draw solute permeation in forward osmosis: modeling and experiments, Environ. Sci. Technol. 44 (2010) 5170-5176.

[41] P. van de Witte, P.J. Dijkstra, J.W.A. van den Berg, J. Feijen, Phase separation processes in polymer solutions in relation to membrane formation, J. Membrane Sci. 117 (1996) 1-31. 
[42] R.W. Baker, Membrane Technology and Applications, second ed., J. Wiley, Chichester, New York, 2004.

[43] J.R. McCutcheon, M. Elimelech, Influence of membrane support layer hydrophobicity on water flux in osmotically driven membrane processes, J. Membrane Sci. 318 (2008) 458-466.

[44] M.T.M. Pendergast, J.M. Nygaard, A.K. Ghosh, E.M.V. Hoek, Using nanocomposite materials technology to understand and control reverse osmosis membrane compaction, Desalination 261 (2010) 255-263.
[45] L. Shi, S.R. Chou, R. Wang, W.X. Fang, C.Y. Tang, A.G. Fane, Effect of substrate structure on the performance of thin-film composite forward osmosis hollow fiber membranes, J. Membrane Sci. 382 (2011) 116-123.

[46] J. Wei, X. Liu, C.Q. Qiu, R. Wang, C.Y.Y. Tang, Influence of monomer concentrations on the performance of polyamide-based thin film composite forward osmosis membranes, J. Membrane Sci. 381 (2011) 110-117. 